\title{
STRAWBERRY ALGORITHM AND ITS NEWLY EVOLVED VARIANT FOR PATTERN NULLING IN AN ANTENNA ARRAY
}

\author{
Subhashini R. Konidala*
}

\begin{abstract}
In this article attempt has been made for the first time to apply the reported strawberry optimization technique to antenna array synthesis problem. The algorithm is further modified by reinforcing it with adaptive values for the two key parameters known as runner length and root length embedded in the mathematical expression governing the movement of the mother plant from one position to another in the search space to locate the optimum solution. The case studies cited here refer to linear and circular array configurations. The design constraints are limited to minimizing the side lobe level and restricting the first null beam width, which play significant roles in antenna array performances. The important features which greatly influence in achieving the said objectives are either placement of antenna elements or amplitudes of excitations of these elements or both. And the recently reported nature inspired metaheuristic optimization algorithms have addressed to these antenna problems quite effectively and the application of the strawberry algorithm for the first time and the unraveling of a new algorithm known as reduced step size strawberry algorithm (rss-SBA) a variant of the existing SBA have shown quite exciting results thus opening avenues for these techniques to be the potential contenders in the race of authenticating their positions in the domain of nonlinear function optimization.
\end{abstract}

Mathematics Subject Classification. 00.XX.

Received December 26, 2018. Accepted May 2, 2020.

\section{INTRODUCTION}

This piece of research work is primarily aimed at establishing the superiority of the proposed Strawberry optimization algorithm (SBA) and its new variant rss-SBA and much emphasis is given to validate the claim when those are applied to the antenna array synthesis problem. Recently, nature inspired metaheuristic optimization techniques have gained significant importance in solving non-linear problems quite successfully in comparison to their conventional counterparts based on the error derivative techniques. However introduction of evolutionary algorithms are truly the game changers in such issues. These concepts led to the development of many efficient and powerful metaheuristic algorithms to effectively handle the situation with encouraging results. The success story has created a great impact which has strongly motivated the researchers to evolve new algorithms and there has been a relentless effort in this direction with great spirit and enthusiasm with each innovative technique superseding the other. Being triggered by such philosophy and fascinated by the exciting results of various

Keywords. Strawberry algorithm, metaheuristic algorithms, antenna array, optimization, nature inspired techniques.

Department of EE/NIT Rourkela, Rourkela, India.

* Corresponding author: subhashiniratna@gmail.com 
nature-inspired metaheuristic optimization algorithms, researchers have successfully applied these to antenna array problems and achieved enhanced performances $[2,9,13,20,25,28,50]$. The continuous pursuit of researchers to develop a new algorithm is always a challenge and the undeterred endeavor has succeeded in evolving a new technique known as strawberry algorithm (SBA) [29]. This algorithm has been successfully applied to many engineering fields such as control, energy etc. $[7,8,22,23]$. The present author attempted to carry out exhaustive studies to determine the worthiness of SBA applied to antenna array synthesis i.e. to design the inter element spacings and their excitation amplitudes. And accordingly the strawberry algorithm as an optimization tool has been applied to antenna array for the first time and it resulted in a faster convergence characteristic in addition to remarkable improvement in sidelobe level suppression and placement of nulls at prescribed locations. To name a few nature inspired optimization algorithms applied to antenna array synthesis are genetic algorithm (GA) [2, 9, 50], differential evoultion (DE) [6,11,12, 14, 32, 33, 47, 52], particle swarm optimization (PSO) $[12,24,25]$, ant colony optimization (ACO) [38], simulated annealing (SA) [39], bacterial foraging optimization (BFO) [13], firefly algorithm (FA) [45,51], cuckoo search (CS) [48], harmony search (HS) [17], invasive weed optimization (IWO) [18,21,34,35,41], back tracking search algorithm (BSA) [10,19], flower pollination algorithm (FPA) [42], bee colony algorithm (BCA) [16,26], grey wolf optimization (GWO) [44], cat swarm optimization (CSO) [36,37], antlion optimization (ALO) [31], spider monkey optimization (SMO) [49], biogeography based optimization (BBO) $[3,4,46,47]$, taguchi method (TM) [11] and other techniques etc. Being inspired by such list of algorithms which have delivered quite significant results, the present author determined to modify the existing SBA [29] to develop a novel technique. The concept proposed has been logically explained. The results obtained definitely speaks in favor of the proposed method. In order to incorporate the changes as suggested, the two parameters, runner length $d_{\text {runner }}$ and root length $d_{\text {root }}$ are made to vary continuously with each iteration. This involves in exponential decaying these parameters as the iteration process continues. This very idea that germinated in this context discovered a new variant of the original SBA [29], known as reduced step size SBA (rss-SBA). The current focus in this article is to explore the possibility of identifying strawberry algorithm (SBA) as well as its variant (rss-SBA) as a successor in finding optimal solutions in nonlinear problems, with particular emphasis to antenna array synthesis. And in fact the results obtained have clearly paved the way for not only the strawberry algorithm (SBA) but also for the proposed rss-SBA. The rest of the paper is organized in the following way. Section 2 presents an overview of the so called strawberry algorithm and the development of the proposed rss-SBA followed by Section 3, where the case study to fix $\gamma$ value is discussed. Validation using some benchmark functions are shown in Section 4. Section 5 is exclusively dedicated in experimenting the technique on the antenna array pattern synthesis. And finally conclusions are drawn as given in the Section 7.

\section{An OVERVIEW OF STRAWBERRY ALGORITHM [29]}

The essential components ingrained with this nature inspired algorithm are global search as well as local search abilities. These combined strategies have led to the evolution of a powerful optimization concept where the chances of the solution reaching the global optima are very high. Both the exploration and exploitation in the search space for finding an optimum solution are achieved using global and local searches respectively. The basic idea in relation to strawberry plant taking part in optimization procedure lies with this fact that it comprises both runners and roots, the two most powerful integral parts which decide the course of action i.e. whether convergence is guaranteed leading to optimal solution or not. While the runners play a significant role in global search as they have a greater reach, the roots dedicate themselves in local searches because they tend to move in small steps around a given domain to find the solution. And the process of finding the objective continues in each iteration with every mother plant giving birth to a daughter plant carrying the same characteristics to maintain the legacy. The runners and roots belonging to a plant act as active agents to accomplish their mission and in doing so the mother plants sacrifice their lives for the survival of the daughter plants, which form the fundamental principle of evolution i.e. a new generation is formed with the daughter plants taking over the batten in the race of survival from the mother plants. This ordeal, which is a perpetual struggle to find food 


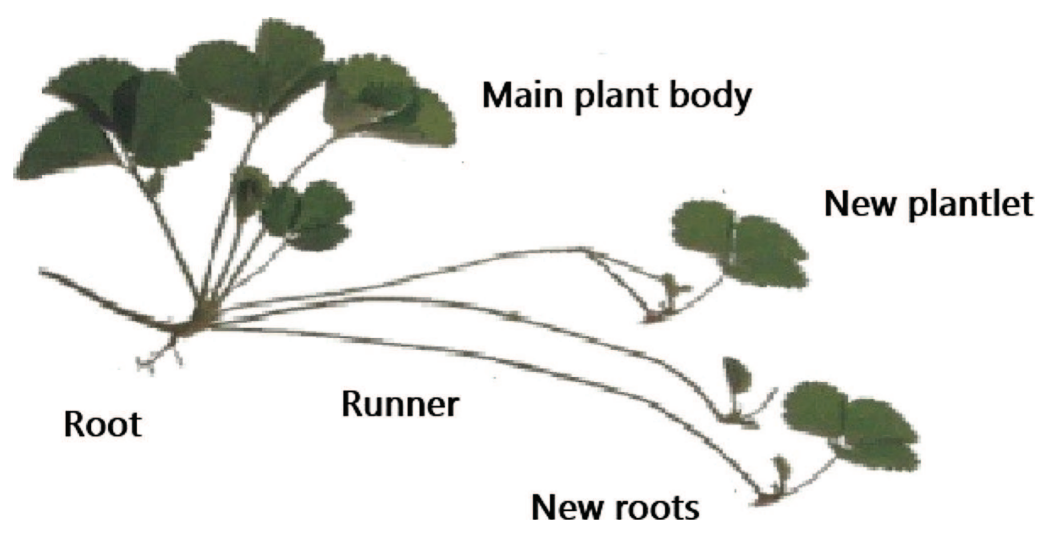

FiguRe 1. Strawberry plant.

for the very existence of the plant mimics a process of optimization. A typical strawberry plant is shown in Figure 1. The accompanied flowchart in Figure 2 clearly depicts the process involved in it.

\subsection{Mathematical modeling of SBA [29]}

The location of the runner and roots are contained in the following matrix form

$$
\begin{aligned}
\bar{X}_{\text {prop }}(i)= & {\left[\bar{X}_{\text {runner }}(i), \bar{X}_{\text {root }}(i)\right]=[\bar{X}(i), \bar{X}(i)] } \\
& +\left[d_{\text {runner }} \bullet \bar{r}_{1}(i), d_{\text {root }} \bullet \bar{r}_{2}(i)\right] .
\end{aligned}
$$

Further, $\bar{r}_{1}, \bar{r}_{2} \in \mathbb{R}^{m \times n}$ are random matrices whose elements are independent random numbers with uniform distribution in the range $[-0.5,0.5], d_{\text {runner }}$ and $d_{\text {root }}$ are two scalars denoting the weighting factors for runners and roots of the mother plant respectively which influence the amount of the movement of the mother plant for the next generation (iteration) in search of the desired goal. Generally $d_{\text {runner }} \gg d_{\text {root }}$ as $d_{\text {runner }}$ controls the exploration and $d_{\text {root }}$ takes care of exploitation. Thus the matrix $\bar{X}_{\text {prop }}(i)$ indicates the plant propagation matrix at $i$ th iteration is of the order $m \times 2 N$ which represents the existence of both runners and roots in one matrix. Here $N$ represents the number of mother plants and $m$ signifies the number of variables to be optimized. Both $\bar{X}_{\text {runner }}(i)$ and $\bar{X}_{\text {root }}(i)$ matrices are of order $m \times N$.

\subsection{Development of the proposed rss-SBA and its mathematical treatment}

The fundamental concept that triggered to develop this novel algorithm is based on the fact that if an optima is guaranteed for a problem under test then the solution vector in an optimization process will definitely move towards it. This explains the phenomenon that the search space is gradually shrinking as the iterative process continues to proceed. Thus it motivated the author to explore means of reducing the $d_{\text {runner }}$ and $d_{\text {root }}$ dynamically and to link the same with the iteration. In this method a common strategy is adopted where an exponential reduction technique was conceived.

$$
\begin{aligned}
d_{\text {runner }}(i) & =\left.d_{\text {runner }}\right|_{\text {intitial }} \exp (-\gamma i / \text { itmax }) \\
d_{\text {root }}(i) & =\left.d_{\text {root }}\right|_{\text {intitial }} \exp (-\gamma i / \text { itmax }) .
\end{aligned}
$$

Here itmax represents the maximum number of iterations used, the values of the $d_{\text {runner }}$ and $d_{\text {root }}$ are continuously reduced from a initial set of values. And equation (2.1) representing the original SBA is now modified as shown below

$$
\bar{X}_{\text {prop }}(i)=[\bar{X}(i), \bar{X}(i)]+\left[d_{\text {runner }}(i) \bullet \bar{r}_{1}(i), d_{\text {root }}(i) \bullet \bar{r}_{2}(i)\right]
$$




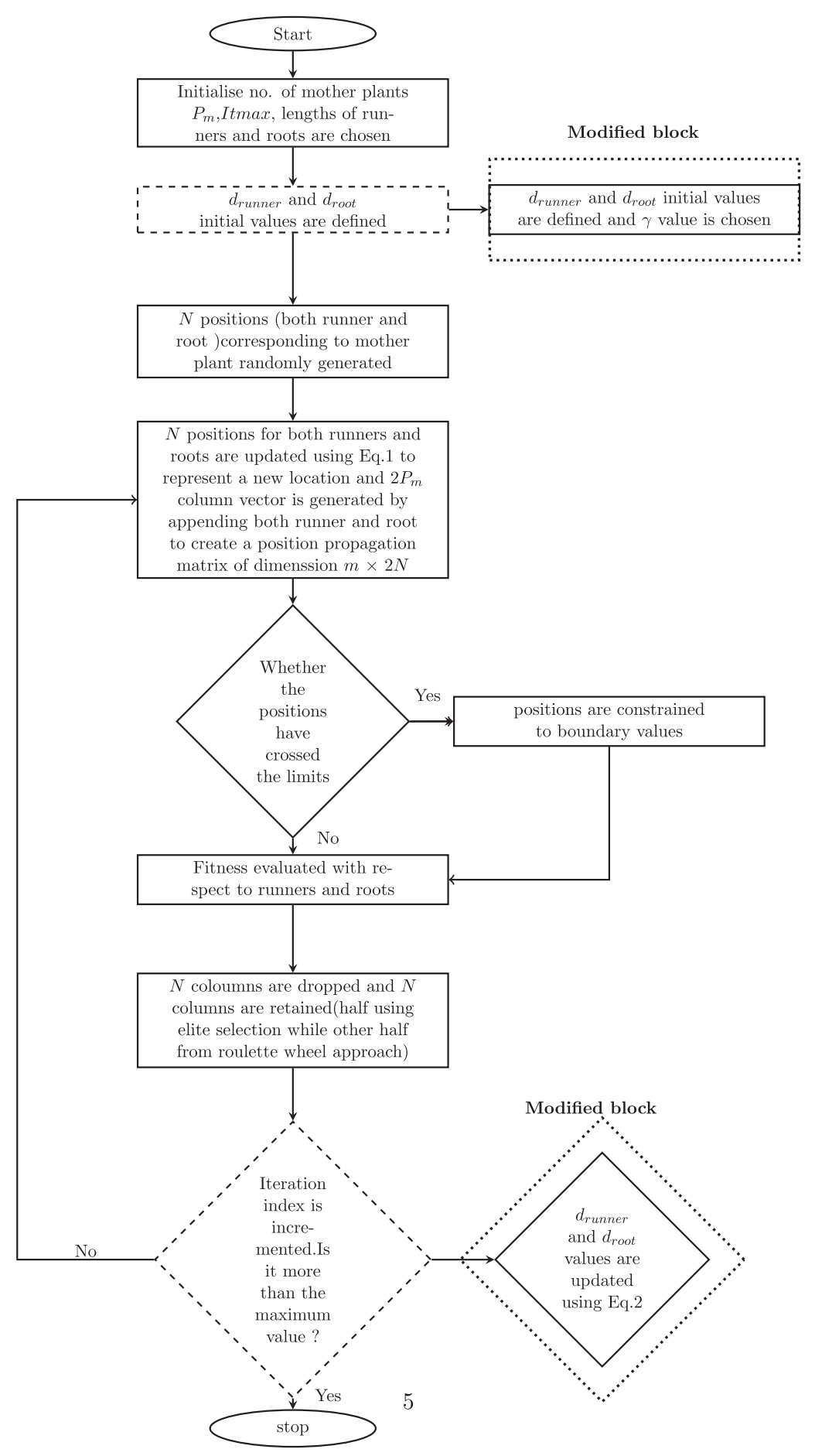

FiguRE 2. Flowchart of the conventional SBA and rss-SBA. 
TABle 1. Pseudo Code of SBA [29] Algorithm.

Step 1. The no. of mother plants $P_{m}=N$, no. of variables $m$, iteration Itmax, $d_{\text {runner }}$ and $d_{\text {root }}$ values are specified.

Step 2. Mother plants (for both runners $\bar{X}_{\text {runner }}$ and roots $\bar{X}_{\text {root }}$ independently) of dimension $m \times N$ is randomly generated (initialized).

Step 3. Iteration process starts and a plant propagation matrix $\bar{X}_{\text {prop }}$ is updated using equation (2.1) of dimension $m \times 2 N$ by appending both $\bar{X}_{\text {runner }}$ and $\bar{X}_{\text {root }}$.

Step 4. The position matrix $\bar{X}_{\text {prop }}$ is verified with respect to the boundary limits assigned to the variables to be optimized. If the positions have crossed the refined limits then the boundary value will be assigned to the variable else the updated positions will be retained.

Step 5. Then using two different strategies, i.e. elite selection method and roulette wheel approach on the $\bar{X}_{\text {prop }}$ matrix, $N / 2$ columns are dropped one after another. The $\bar{X}_{\text {prop }}$ will now have a shape of $m \times N$ healthy solutions.

Step 6. The $\bar{X}_{\text {runner }}$ and $\bar{X}_{\text {root }}$ are again reset to the just computed $\bar{X}_{\text {prop }}$ of size $m \times N$.

Step 7. If termination condition is achieved (i.e. iteration count if it exceeds Itmax), then process stop yielding the so called optimal result else the process repeats by going to Step 3 again by increasing the iteration count.

TABle 2. Pseudo Code of the proposed reduced step size version (rss-SBA).

Steps 1-7. The same procedure is adopted as given in the Table 1.

Step 8. Before the termination criteria is validated, the $d_{\text {runner }}$ and $d_{\text {root }}$ which have been assigned initial values will now be modified using the equation $(2.2)$ so that when the next iteration starts, the $d_{\text {runner }}$ and $d_{\text {root }}$ will have reduced value with reference to the earlier one. The rest of the process is same as the convention SBA described in Table 1.

Further $\gamma$ is a constant coefficient which controls the rate of change of both the runner and root values exponentially. It is quite obvious that when the solution starts to move towards the global optima all the search agents(mother plants) will converge too. This indicates and authenticates that the search space In fact shrinks for achieving optimal results. Thus the fixed values of $d_{\text {runner }}$ and $d_{\text {root }}$ as already mentioned in the conventional SBA may not reach the true optima causing a prematured convergence. However the present technique which employs a reducing step size (reducing the value with increase in the iteration count) concept takes care of such eventuality. The flowchart for the conventional SBA and the rss-SBA is given in the Figure 2 (Tabs. 1 and 2).

\section{Study on PARAMETER $\gamma$ USED IN THE PROPOSED ALGORITHM (RSS-SBA)}

In order to find out the $\gamma$ value different case studies on unimodal, multimodal functions in Table 3 are performed. The parametric setup comprises of, population size $=50$, dimension $=30$, number of iterations are 1000. $\left.d_{\text {runner }}\right|_{\text {intitial }}$ is set to be equal to the range of the function i.e. [Upper bound - Lower bound]. $\left.d_{\text {root }}\right|_{\text {intitial }}$ is taken as $10 \%$ of [Upper bound - Lower bound]. The $\gamma$ value is picked up on the basis of least fitness value obtained by the algorithm for the cost function. In Figure 3, the $\gamma$ value of 36 gives a minimum value of the cost function which is a close optimum solution of the function. The $\gamma$ value is set after performing 15 independent trials on the function $F_{1}$.

The study is repeated for $F_{2}$ with the rss-SBA parameters set to population size 50 and iterations as 1000 with the $\left.d_{\text {runner }}\right|_{\text {intitial }}$ and $\left.d_{\text {root }}\right|_{\text {intitial }}$ fixed as discussed prior is shown in Figure 4 . The least fitness value 
TABLE 3. Benchmark functions [27] used to validate the proposed rss-SBA algorithm.

\begin{tabular}{llll}
\hline \hline Benchmark test functions & $D$ & Range & Optimum \\
\hline$F_{1}=\sum_{i=1}^{N}\left(x_{i}\right)^{2}$ & 30 & {$[-100100]$} & 0 \\
$F_{2}=\sum_{i=1}^{N}\left|x_{i}\right|+\prod_{i=1}^{N}\left|x_{i}\right|$ & 30 & {$[-10,10]$} & 0 \\
$F_{3}=\sum_{i=1}^{N}\left[100\left(x_{i+1}-x_{i}{ }^{2}\right)^{2}+\left(1-x_{i}{ }^{2}\right)\right]$ & 30 & {$[-30,30]$} & 0 \\
$F_{4}=\sum_{i=1}^{N}\left(\sum_{j=1}^{i} x_{i}\right)^{2}$ & 30 & {$[-100,100]$} & 0 \\
$F_{5}=\sum_{i=1}^{N}\left[x_{i}{ }^{2}-10 \cos 2 \pi x_{i}+10\right]$ & 30 & {$[-5.12,5.12]$} & 0 \\
$F_{6}=-20 \exp \left(-0.2 \sqrt{\frac{1}{n} \sum_{i=1}^{N} x_{i}{ }^{2}}\right)-\exp \left(\frac{1}{n} \sum_{i=1}^{N} \operatorname{con} 2 \pi x_{i}\right)+20+e$ & 0 \\
$F_{7}=\left[\frac{1}{500}+\sum_{j=1}^{25} \frac{1}{j+\sum_{i=1}^{2}\left(x_{i}-a_{i i}\right)^{6}}\right]^{-1}$ & 30 & {$[-20,20]$} \\
$F_{8}=\left(x_{2}-\frac{5.1}{4 \pi^{2}} x_{1}{ }^{2}+\frac{5}{\pi} x_{1}\right)^{2}+10\left(1-\frac{1}{8 \pi} \cos x_{1}+10\right)$ & 30 & {$[-65.53,65.53]$} & 0.998004 \\
$F_{9}=\frac{1}{4000} \sum_{i=1}^{N} x_{i}{ }^{2}-\prod_{i=1}^{N} \cos \left(\frac{x i}{\sqrt{i}}\right)+1$ & 30 & {$[-5,10] *[-0,15]$} & 0.398 \\
$F_{10}=\sum_{i=1}^{N} i . x_{i}{ }^{4}+\operatorname{random}[0.1]$ & 30 & {$[-600,600]$} & 0 \\
& 30 & {$[-1.28,1.28]$} & 0 \\
\hline
\end{tabular}

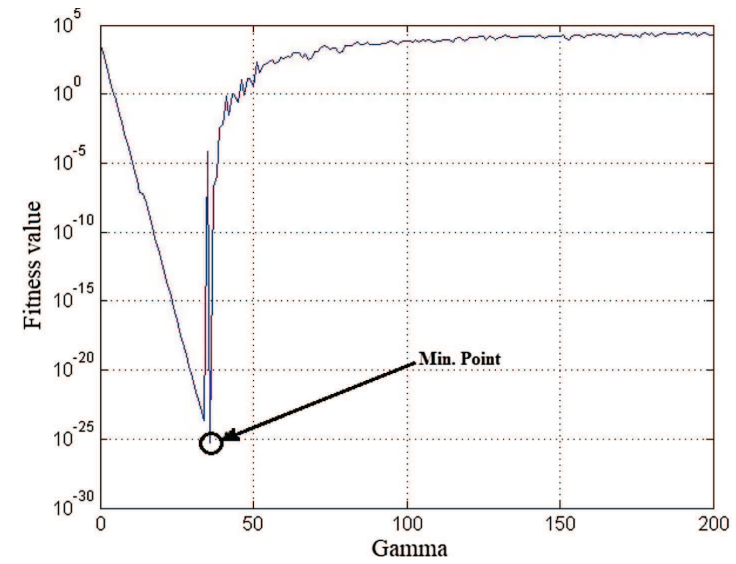

Figure 3. $\gamma$ fixation plot for the function $F_{1}$.

is obtained at $\gamma$ value of 26 . The experiment is continued for 15 independent trials. Figure 5 shows that the optimal solution of the function $F_{5}$ reaches at a $\gamma$ value of 20. Figure 6 gives the discrete value of $\gamma$ as 10 for $F_{7}$ that is leading to the optimal solution of the function.

Also another important observation on various case studies (not reported in the document) revealed that, with the increase in the dimension value, keeping the iteration count constant it is observed that the $\gamma$ value decreases. A detailed study on movement of the runners and roots in the bounded search space of Rastrigin and Ackley function [27] is carried out in the next section with $\gamma$ value as 4 .

\section{VAlidation of SBA AND RSS-SBA in CLASSiCAL BENCHMARK FUnCtions [27]}

In order to prove that the SBA algorithm as well as its variant rss-SBA are capable of solving the optimization problem two bench mark functions are analyzed in detail with SBA and rss-SBA. 


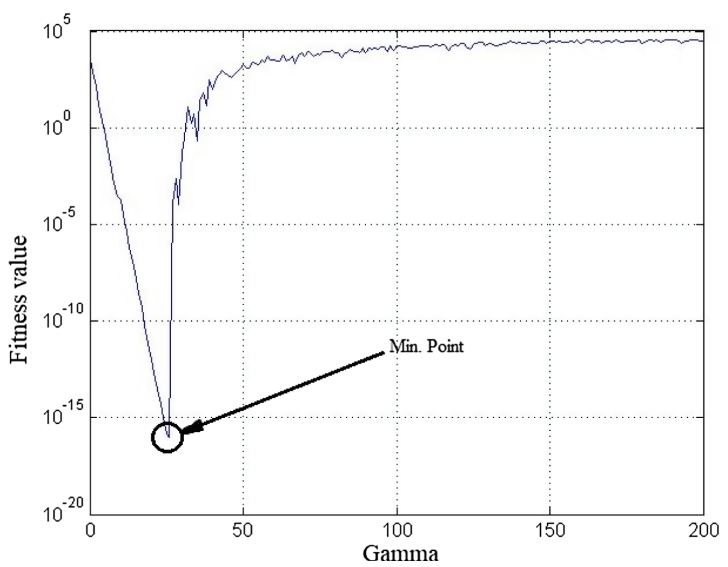

FiguRE 4. $\gamma$ fixation plot for the function $F_{2}$.

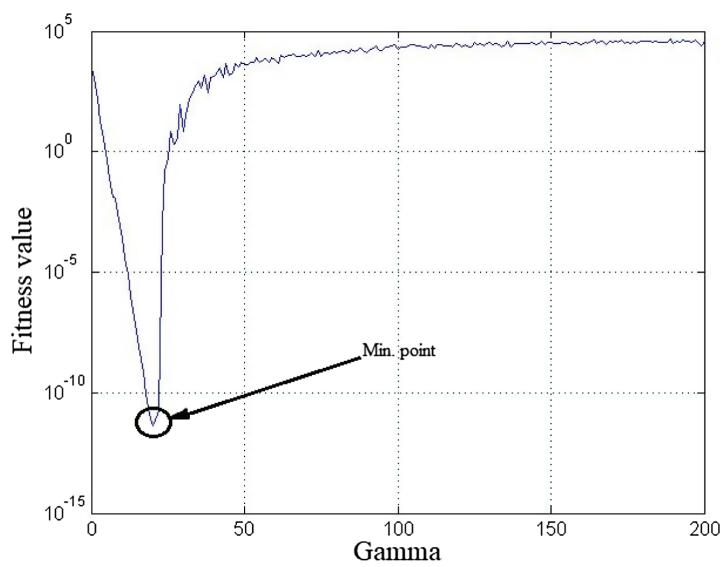

FiguRE 5. $\gamma$ fixation plot for the function $F_{5}$.

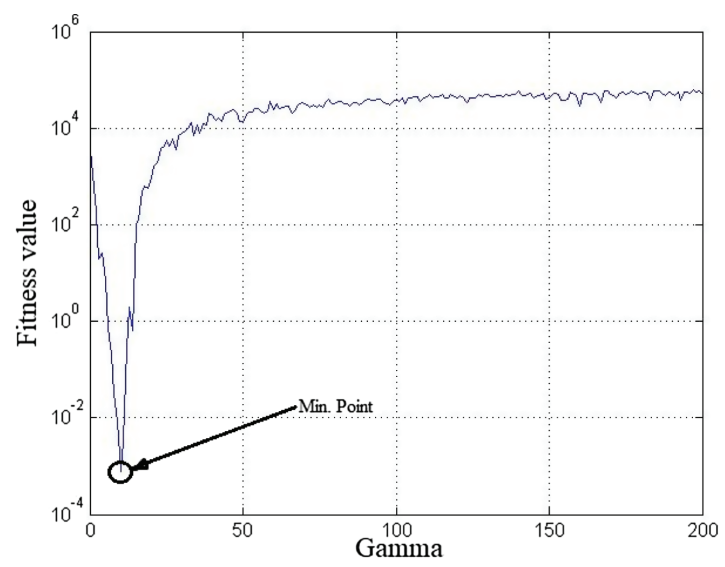

FiguRE 6. $\gamma$ fixation plot for the function $F_{7}$. 


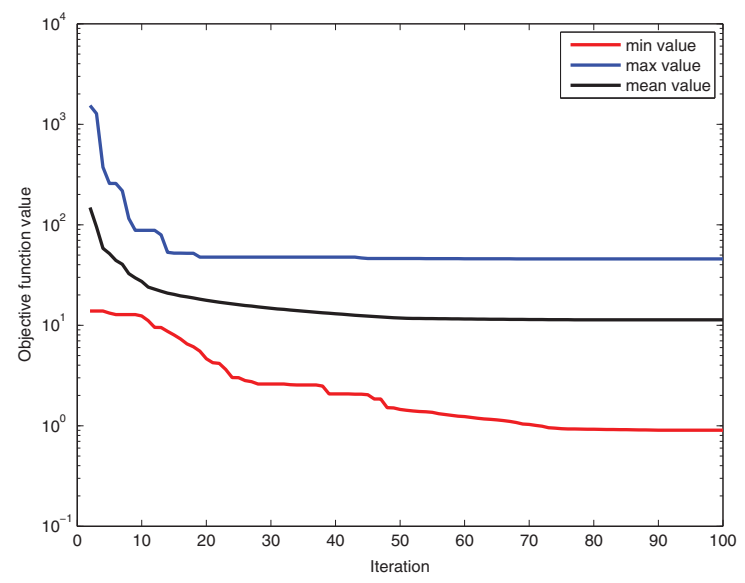

(a)

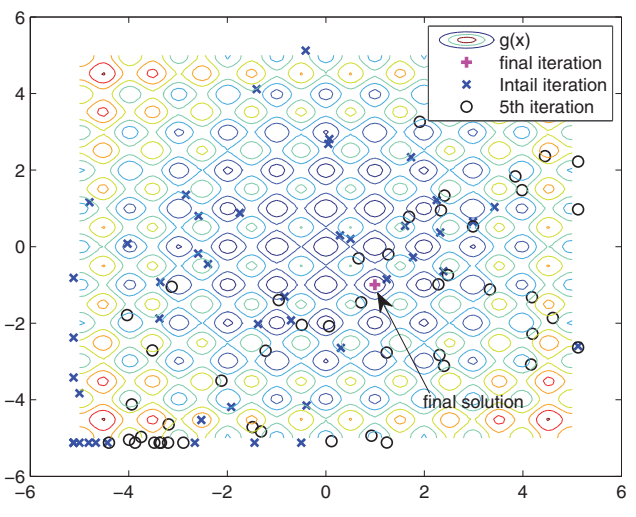

(b)

FiguRE 7. Rastrigin function. (a) Convergence curves of min, mean and max values using SBA.

(b) Locations of mother plants for SBA in the simulation of two dimensional space.

\subsection{Case study on $N$-dimensional Rastrigin function [27]}

$$
f(x)=\sum_{i=1}^{N}\left[x_{i}^{2}-10 \cos 2 \pi x_{i}+10\right]
$$

where $x_{i}=\in[-5.12,5.12](i=1,2, \ldots, N)$. The function has a global minimum at $x=0$. The parameters chosen for both the algorithms are population size as $P_{m}=30 ; N=2 ; d_{\text {runner }}=2.0 ; d_{\text {root }}=0.3$ and $\gamma=4$. The $\gamma=4$ is chosen after a series of experiments. The proposed rss-SBA as well as original SBA are executed for 15 independent trials comprising of 100 iterations each. The convergence characteristics of min, mean and max values of the function is shown in the Figure 7a which are averaged over 15 trials. It is inferred from this characteristic that the SBA is very fast in convergence as its min value is settling down at around a floor level of magnitude one from 70th iteration onwards. The positions of the mother plants at various instants of time index i.e. at an initial point of 5th iteration and at the final iteration count are shown in Figure 7b when the conventional SBA [29] is applied on the Rastrigin function. From the contour plot it is also observed that as the iteration process continues, the search agents (mother plants) start to congregate from an absolute random scattered distribution over the entire search space (initial iteration) to a narrow area clearly suggesting that the solution vector is in right track. Similarly when the proposed rss-SBA is applied to validate its ability to perform, it is evident from the convergence characteristics shown in the Figure 8a that the proposed method too has a very fast response. Further, it is also seen that the proposed rss-SBA the min value of the objective function settles much below (at around 0.1) than that of using SBA. Another important interpretation was made that in order to achieve the same floor level of one as in case of SBA, the proposed rss-SBA does it in around 10 iterations only to prove its effectiveness. It also consolidates the fact that the locations of the mother plants during the transition phases at various time instants using rss-SBA are much closer to optimal values in comparison to SBA as shown in the Figure 8b.

\subsection{Case study on a $N$-dimensional Ackley function [27]}

$$
g(x)=-20 \exp \left(-0.2 \sqrt{\frac{1}{n} \sum_{i=1}^{N} x_{i}{ }^{2}}\right)-\exp \left(\frac{1}{n} \sum_{i=1}^{N} \cos 2 \pi x_{i}\right)+20+e,
$$

where $x_{i}=\in[-20,20](i=1,2, \ldots, N)$. 


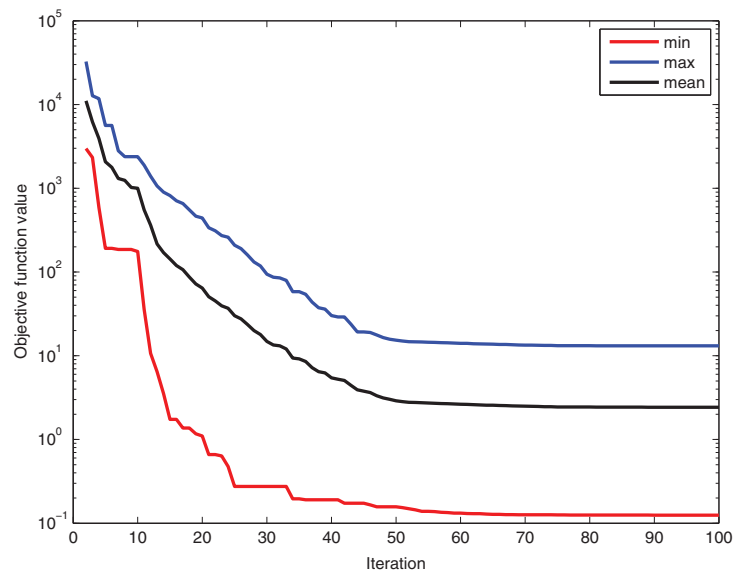

(a)

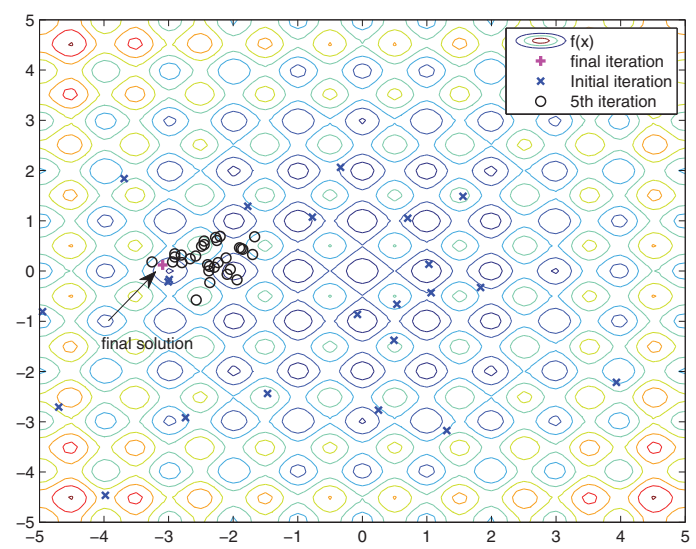

(b)

FIgURE 8. Rastrigin function. (a) Convergence curves of min, mean and max values using rssSBA. (b) Locations of mother plants for rss-SBA in the simulation of two dimensional space.

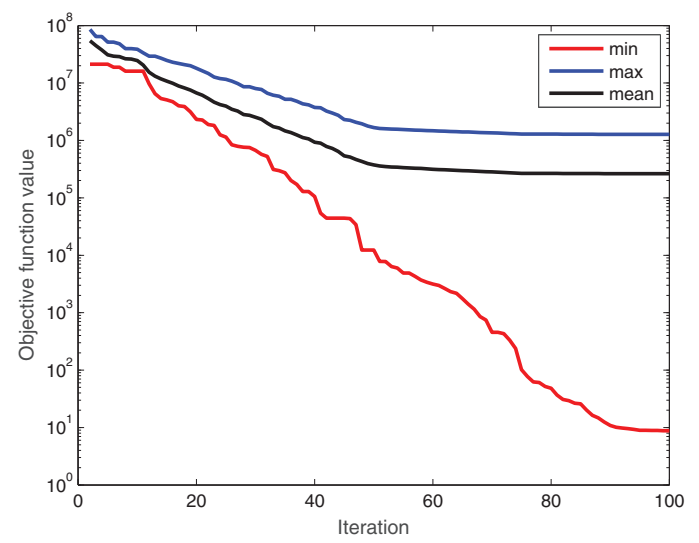

(a)

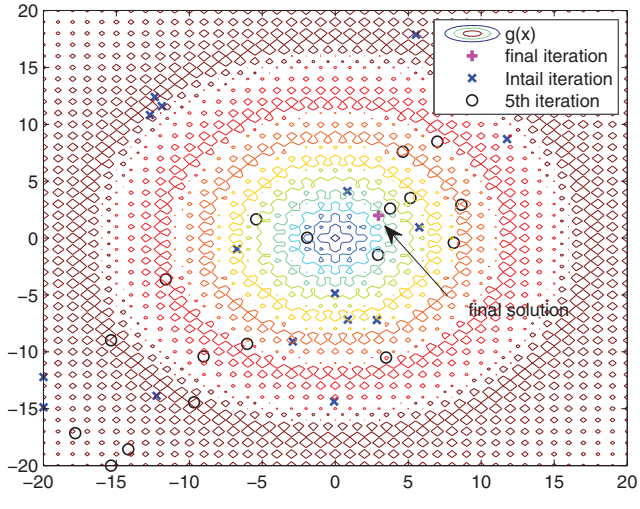

(b)

Figure 9. Ackley function. (a) Convergence curves of min, mean and max values using SBA.

(b) Locations of mother plants for SBA in the simulation of two dimensional space.

Another function having a global minimum at $x=0$ is also studied. The SBA algorithm is applied to this function and was run for 100 iterations. In order to choose the best one, 15 independent trials are conducted. The convergence characteristics of min, mean and max values of the objective function is shown in the Figure 9a. With a population size as $P_{m}=30 ; d_{\text {runner }}=2.5 ; d_{\text {root }}=0.4 ; N=2$ and $\gamma=4$ the simulation study is carried out. The location of the mother plants at various iterations are shown in the Figure 9b. While the results for this function is analyzed it is observed that the min value of the objective function settles at a level having a magnitude of 10 using SBA (Fig. 9a) which completely differs to that obtained using the proposed rss-SBA (Fig. 10a), where the settling floor level is around 0.1. In fact it is a huge performance gain. Similar behavior of improvement in results is also witnessed when the location of the mother plants using SBA (Fig. 9b) is compared with those results obtained using rss-SBA (Fig. 10b). It is clearly visible that the search agents have started to 


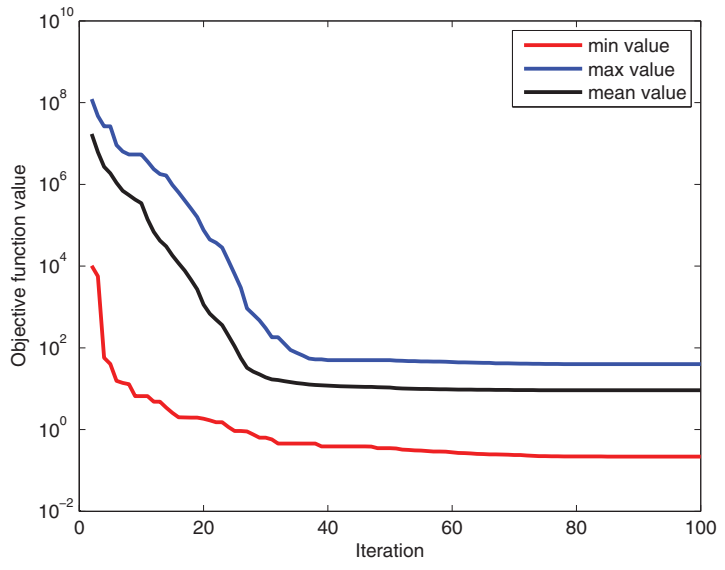

(a)

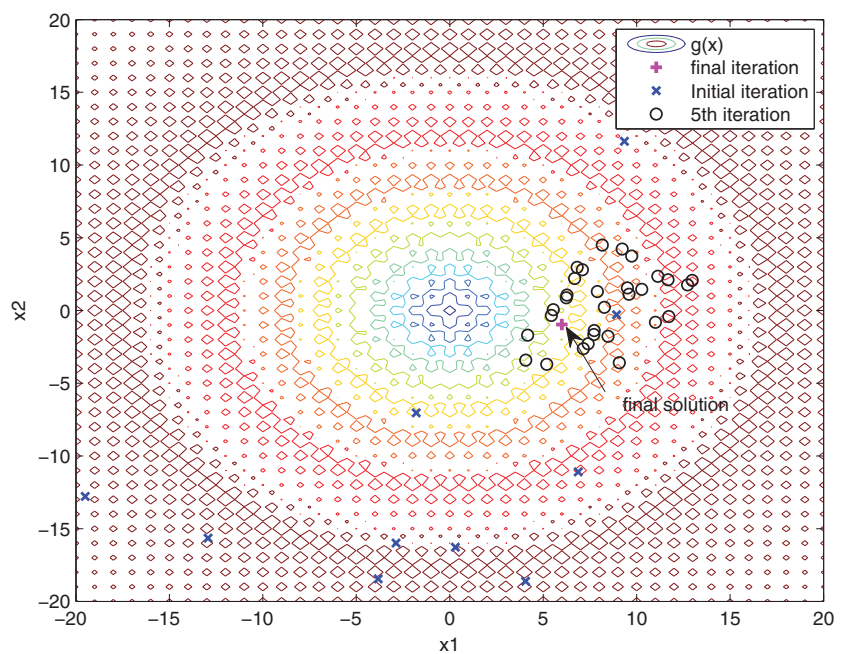

(b)

Figure 10. Ackley function. (a) Convergence curves of min, mean and max values using rssSBA. (b) Locations of mother plants for rss-SBA in the simulation of two dimensional space.

form a close congregation only at 5th iteration (using rss-SBA) which is way ahead compared to the response of SBA where agents are still dispersed.

The inference that is almost drawn from the case studies of Rastrigin and Ackely functions is that the proposed rss-SBA has a tremendous potential to solve the optimization of nonlinear functions quite effectively.

\subsection{Comparison with other metaheuristic algorithms}

The encouraging results obtained from the two benchmark problems opened the avenues to further validate the robustness of SBA algorithm when applied to other benchmark functions. Table 4 represents two key perform indicators known as average and standard deviation when optimization was carried out for the test functions based on 15 independent runs. The results depicted in the Table 4 clearly places the proposed rss-SBA as a true competitor in comparison to SBA and other meta heuristic algorithms. These motivated the author to apply this nature inspired technique to antenna array synthesis problems. The main aim was to investigate how efficiently the antenna array problems can be solved, particularly designing the element spacing and/or the antenna element excitations as the case may be for both linear and circular topologies using SBA and the proposed rss-SBA. Application of the conventional SBA has also not been reported till date in the antenna domain. Thus attempt has been made to analyze antenna synthesis using both SBA [29] and the proposed rss-SBA approaches.

\section{Application of strawberry algorithm [29] And proposed RSS-SBA to Antenna ArRay SYNTHESIS}

In order to further expand the domain of application, the antenna array synthesis problem is investigated. In the present context, this study is exclusively divided into two categories: linear antenna array (LAA) and circular antenaa array (CAA). These two topologies have been independently analyzed. 
TABLE 4. Results of various algorithms applied to unimodal and multi modal benchmark functions $(D=30)[30]$.

\begin{tabular}{|c|c|c|c|c|c|c|c|c|}
\hline \multirow[t]{2}{*}{ Function name } & \multicolumn{2}{|c|}{ rss-SBA } & \multicolumn{2}{|c|}{ SBA } & \multicolumn{2}{|c|}{$\mathrm{PSO}$} & \multicolumn{2}{|l|}{ SMS } \\
\hline & ave & std & ave & std & ave & std & ave & std \\
\hline Sphere $F_{1}$ & $8.3787 \mathrm{e}-29$ & $1.9109 \mathrm{e}-15$ & $8.70 \mathrm{e}-27$ & $1.70 \mathrm{e}-14$ & $2.70 \mathrm{e}-09$ & $1.00 \mathrm{e}-09$ & 0.056987 & 0.014689 \\
\hline Schwefel1 $F_{2}$ & $2.2523 \mathrm{e}-17$ & $7.3860 \mathrm{e}-19$ & $2.54 \mathrm{e}-16$ & $8.75 \mathrm{e}-18$ & $4.71 \mathrm{e}-06$ & $1.49 \mathrm{e}-06$ & 0.959865 & 0.82345 \\
\hline Rosenbrock $F_{3}$ & 0.0088 & 0.0035 & 0.010247 & 0.001045 & 0.123401 & 0.216051 & 0.085348 & 0.140149 \\
\hline Schwefel1 $F_{4}$ & $-7.3092 \mathrm{e} 03$ & 29.0285 & -3098.48 & 301.8919 & -1367.01 & 146.4089 & -4.20735 & $9.36 \mathrm{e}-16$ \\
\hline Rastrigin $F_{5}$ & $5.6843 \mathrm{e}-12$ & $6.7858 \mathrm{e}-10$ & $1.14 \mathrm{e}-11$ & $6.64 \mathrm{e}-09$ & 0.278588 & 0.218991 & 1.32512 & 0.326239 \\
\hline Ackley $F_{6}$ & $7.9048 \mathrm{e}-14$ & $1.6589 \mathrm{e}-15$ & $2.6709 \mathrm{e}-12$ & $3.6987 \mathrm{e}-11$ & $1.11 \mathrm{e}-09$ & $2.39 \mathrm{e}-11$ & $8.88 \mathrm{e}-06$ & $8.56 \mathrm{e}-09$ \\
\hline De jong $F_{7}$ & $3.0794 \mathrm{e}-04$ & 0.1671 & 4.1498 & 0.0209 & 162.9144 & 119.2351 & 873.7522 & 9.716179 \\
\hline Branin $F_{8}$ & 0.6124 & 2.7085 & 0.2036 & 3.437 & 450.0688 & 157.8496 & 899.8578 & $1.99 \mathrm{e}-05$ \\
\hline Griewank $F_{9}$ & $2.0716 \mathrm{e}-08$ & $2.6089 \mathrm{e}-09$ & $2.59 \mathrm{e}-06$ & 0.000448 & 0.273674 & 0.204348 & 0.70609 & 0.907954 \\
\hline \multirow[t]{3}{*}{ Quartic $F_{10}$} & $6.3795 \mathrm{e}-04$ & 0.0016 & 0.39788 & 0.612578 & 0.001398 & 0.001269 & 0.000304 & 0.000258 \\
\hline & \multicolumn{2}{|l|}{ FPA } & \multicolumn{2}{|c|}{ CS } & \multicolumn{2}{|l|}{$\mathrm{FA}$} & \multicolumn{2}{|c|}{ GA } \\
\hline & ave & std & ave & std & ave & std & ave & std \\
\hline Sphere $F_{1}$ & $1.06346 \mathrm{e}-07$ & $1.27 \mathrm{e}-7$ & $6.50 \mathrm{e}-03$ & $2.05 \mathrm{e}-04$ & 0.039615 & 0.01449 & 0.118842 & 0.125606 \\
\hline Schwefel1 $F_{2}$ & $5.6682 \mathrm{e}-08$ & $3.9 \mathrm{e}-08$ & $2.47 \mathrm{e}-07$ & $2.14 \mathrm{e}-2$ & 0.049273 & 0.019409 & 0.13902 & 0.121161 \\
\hline Rosenbrock $F_{3}$ & 0.781200043 & 0.366891 & 0.007197 & 0.007222 & 2.175892 & 1.447251 & 0.714157 & 0.972711 \\
\hline Schwefel1 $F_{4}$ & -1842.42621 & 50.42824 & -2094.91 & 0.007616 & -1245.59 & 353.2667 & -2091.64 & 2.47235 \\
\hline Rastrigin $F_{5}$ & 0.273294621 & 0.068583 & 0.127328 & 0.002655 & 0.263458 & 0.182824 & 0.659271 & 0.815751 \\
\hline Ackley $F_{6}$ & 0.007398721 & 0.007096 & 8.16e-09 & $1.63 \mathrm{e}-08$ & 0.168306 & 0.050796 & 0.956111 & 0.807701 \\
\hline De jong $F_{7}$ & 18.23309 & 3.074685 & 140.6065 & 92.80327 & 314.4654 & 92.93417 & 95.46331 & 7.163338 \\
\hline Branin $F_{8}$ & 362.0262 & 54.01816 & 401.5247 & 98.16459 & 819.5732 & 109.9663 & 466.3074 & 29.56841 \\
\hline Griewank $F_{9}$ & 0.085021659 & 0.040046 & 0.122678 & 0.049673 & 0.099815 & 0.024466 & 0.487809 & 0.217782 \\
\hline Quartic $F_{10}$ & 0.003105276 & 0.001367 & 0.001321 & 0.000728 & 0.000853 & 0.00504 & 0.010073 & 0.003263 \\
\hline
\end{tabular}

\section{Linear antenaa array (LAA)}

In a linear antenna array configuration comprising of $M=2 N$ elements located along the $x$-axis, the corresponding array factor is governed by the relation [5].

$$
\mathrm{AF}_{l}(\theta)=2 \sum_{q=1}^{N} I_{q} \cos \left(k d_{q} \cos \theta\right)
$$

where $k$ is the wave number represented by $k=2 * \pi / \lambda, \theta$ is the azimuth angle bounded between $-90^{\circ}$ and $90^{\circ}$ or $0^{\circ}$ and $180^{\circ}$ as the case may be (for the sake of comparison with other reported articles) and $d_{q}$ and $I_{q}$ denote the position and the excitation amplitude of the $q$ th antenna element respectively and the phase is assumed to be zero. If the $M$ elements are expressed as $N$ elements, their symmetrical arrangements can be made with $N$ elements in each side centered around the $y$-axis. In order to achieve the desired objective of minimizing the sidelobe level, the array factor $\mathrm{AF}_{l}(\theta)$ is optimized using the so called strawberry algorithm [29] and the proposed rss-SBA to determine the best solutions for antenna element locations or their excitation amplitudes as per the criteria. The results are verified with the recent published techniques like backtrack searching algorithm (BSA) [15], harmony search alogorithm (HSA) [17], cat swarm optimization (CSO) [36], ant lion optimization (ALO) [43], spider monkey optimization (SMO) [1] (Fig. 11).

The problems undertaken in linear antenna array configuration is divided based on two different control strategies: amplitude only control and position only control. The first method is involved in optimizing the excitation amplitudes of antenna elements keeping the inter element spacing $\lambda / 2$ and imposing the constraints of minimizing maximum sidelobe level (MSL) and null depth level (NDL) below a desired specifications. In this category different situations are created to optimize the excitations to be fed to the antenna elements uniformly spaced but exhibiting different characteristics which are considered while the design process is initiated using 


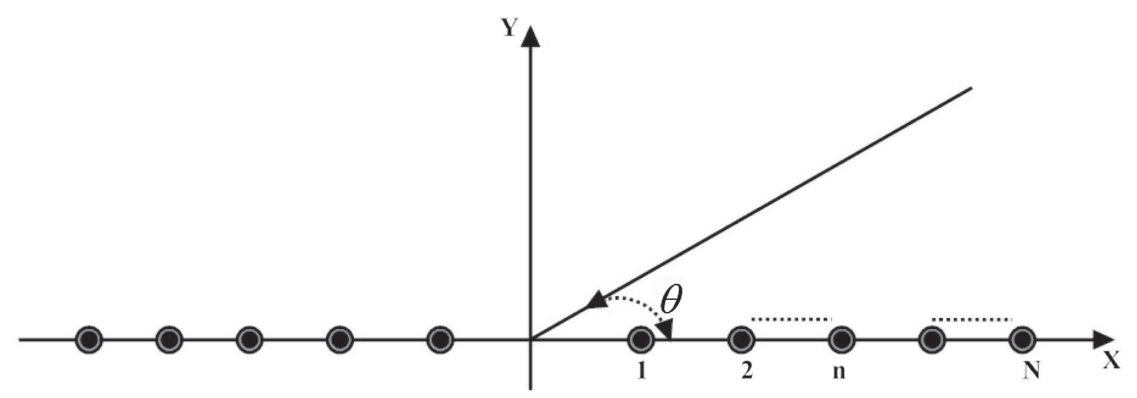

Figure 11. Geometry of $M=2 N$ element symmetric linear array centered around the $y$-axis.

the proposed algorithms. These include wide null, very wide null (specifying a range) and nulls at prescribed locations.

Thus depending upon the requirement, the objective functions have been formulated. For amplitude control mode, the objective function [15] is expressed as

$$
J_{\mathrm{LA}}=\sum_{\theta=\text { lowerlimit }}^{\text {upperlimit }}\left[W \mid \mathrm{AF}_{l o}(\theta)-\mathrm{AF}_{l d}(\theta)\right]+\operatorname{MSLP}
$$

where $J_{\mathrm{LA}}$ is the objective function for linear array amplitude only mode. $\mathrm{AF}_{l o}$ and $\mathrm{AF}_{l d}$ are the array factors using the optimization algorithm and the desired array factor based on Chebyshev criteria respectively. $W$ is the weighting factor associated with the array factor minimization. MSLP is a biased factor related to the maximum sidelobe level. The parameter $W$ plays an important role as it controls the nulls at desired locations with the initial pattern (Chebyshev) imposed elsewhere remain unaltered i.e. the value assigned to $W$ is a user defined value purely based on experience and at prescribed nulls $\operatorname{AF}_{l d}(\theta)=0$ else it is unity. Similarly MSLP known as maximum sidelobe level penalty factor is used in the objective function $J_{\mathrm{LA}}$ to introduce a bias to take care of sidelobe level imposed (from Chebyshev) which is susceptible to change as null have been incorporated to specify a typical requirement. Thus the values of MSLP can be chosen by the user considering a variation of $2 \mathrm{~dB}$ [40] increase over the Chebyshev pattern i.e. the threshold limit is decided as SLL (Chebyshev) $+2 \mathrm{~dB}$ and if MSL crosses this limit MSLP (user defined value) will be added in $J_{\mathrm{LA}}$ else MSLP shall be zero.

The second method deals with optimizing the positions of antenna elements while these are all uniformly excited. These problems considered placement of nulls at desired locations and as well as completely eliminating the effect of the nulls. While position control mode is considered the objective function [24] is chosen and given by

$$
J_{\mathrm{LP}}=\min \left[\max \left(20 \log _{10} \frac{\left|\mathrm{AF}_{l}(\theta)\right|}{\max \left|\mathrm{AF}_{l}(\theta)\right|}\right)\right] .
$$

In this mode while the objective function $J_{\mathrm{LP}}$ is minimized to find the antenna element positions the following constraint is imposed with reference to their inter spacing distance between two consecutive elements. Further, while the objective function is minimized with respect to the sidelobe level, the guiding principles strictly followed as prescribed by Khodier [25] which limits the inter spacing distance are

$$
\left|\left(x_{i}-x_{j}\right)\right|>0.25 \lambda
$$

and

$$
\min \left(x_{i}\right)>0.25 \lambda, \quad i=1,2,3, \ldots, N, \quad i \neq j .
$$




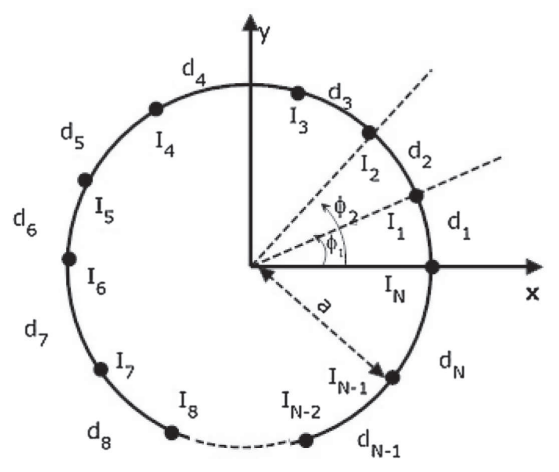

FigURE 12. Circular arrangement of isotropic elements.

\subsection{Circular antenna array (CAA)}

Figure 12 represents another geometrical configuration known as circular antenna array, where the performance greatly depends on the number of elements, spacing between them and their excitation amplitudes. Designing circular antenna array to deliver either a desired or an optimal performance by determining the parameters as stated above is a challenging task. In order to effectively carry out the strategy, the objective function $J_{c}$ is chosen with the limitation that the aperture size is constrained to a particular range depending upon the number of elements [41] and the corresponding array factor is

$$
\operatorname{AF}_{c}(\phi)=\sum_{q=1}^{N} I_{q} e^{j\left(k r \cos \left(\phi-\phi_{q}\right)\right)} .
$$

Here $N$ represents the total number of elements in the circular array. $I_{q}$ and $\phi_{q}$ are the excitation amplitude and angular position of the $q$ th element respectively, $k$ is the wave number given by $2 * \pi / \lambda$ and $r$ is the radius of the circular arrangement. Alternatively $k r$ and $\phi_{q}$ can be described as

$$
k r=2 * \pi * r / \lambda=\sum_{i=1}^{N} d_{i}
$$

$\phi_{q}=2 * \pi / k r \sum_{i=1}^{N} d_{i}$, where $d_{i}$ is the arc length between the $i$ th and $(i-1)$ th elements. The objective function chosen for this example is given by Roy et al. [41]

$$
J_{c}=c_{1} * J_{c}^{\mathrm{NULL}}+c_{2} * J_{c}^{\mathrm{SLA}}+c_{3} * J_{c}^{\mathrm{MSL}}
$$

where,

$$
\begin{aligned}
J_{c}^{\mathrm{NULL}}= & \left|\mathrm{AF}_{c}\left(\phi_{\text {null1 }}\right)\right|+\left|\mathrm{AF}_{c}\left(\phi_{\text {null2 }}\right)\right| \\
J_{c}^{\mathrm{SLA}}= & \frac{1}{\pi+\phi_{\text {null1 }}} \int_{-\pi}^{\phi_{\text {null1 }}}\left|\mathrm{AF}_{c}(\phi)\right| \mathrm{d} \phi \\
& +\frac{1}{\pi-\phi_{\text {null2 }}} \int_{\phi_{\text {null2 }}}^{\pi}\left|\mathrm{AF}_{c}(\phi)\right| \mathrm{d} \phi \\
J_{c}^{\mathrm{MSL}}= & \left|\mathrm{AF}_{c}\left(\phi_{\mathrm{MSL} 1}\right)\right|+\left|\mathrm{AF}_{c}\left(\phi_{\mathrm{MSL} 2}\right)\right| .
\end{aligned}
$$

Further, $\phi_{\text {null1 }}$ and $\phi_{\text {null2 }}$ are the lower and upper nulls (angles) referring to the major lobes respectively. $\phi_{\mathrm{MSL} 1}$ and $\phi_{\text {MSL2 }}$ represent the the angles corresponding to the maximum sidelobe in the lower band from ( $-\pi$ to $\left.\phi_{\text {null1 }}\right)$ 
and upper band from $\left(\phi_{\text {null2 }}\right.$ to $\pi$ ) respectively during the process of optimization. The values $c_{1}, c_{2}$ and $c_{3}$ are weighting factors corresponding to individual objective functions $J_{c}^{\mathrm{NULL}}, J_{c}^{\mathrm{SLA}}$ and $J_{c}^{\mathrm{MSL}}$, respectively where $J_{c}^{\mathrm{NULL}}$ refers to nulls at prescribed locations, $J_{c}^{\mathrm{SLA}}$ indicates average sidelobe levels and $J_{c}^{\mathrm{MSL}}$ describes the maximum sidelobe level. These parameters greatly influence the minimization of the over all objective function $J_{c}$, because varying these parameters add flexibility and bias to different terms embedded in the objective function.

\section{Simulation studies in CASE of Antenna ARRAy}

In order to validate the efficacy of the proposed method the antenna array synthesis problem is considered for both linear and circular array configurations. The sole objective of such studies is to compare the results obtained using rss-SBA with other nature inspired metaheuristic algorithms so that a definite decision can be made to accept it as a worthy alternative. The examples were chosen to compare the performances of various antenna specifications to find either optimized antenna element positions or excitation amplitudes as the case may be subject to a prescribed constraints. And these are mostly comprised of sidelobe level is minimization and placing nulls at desired locations. And such daunting challenges have been adequately taken care of by the SBA and proposed rss-SBA techniques. In order to substantiate the capability of the proposed rss-SBA optimization technique to antenna synthesis problem, the convergence characteristics are plotted. These clearly show that both the conventional SBA [29] and the proposed rss-SBA have very fast responses. And in many cases, rss-SBA has outperformed the SBA not only in speed but converging to minimum settling floor value. In the simulation study the parameters chosen for both SBA and proposed rss-SBA are the number of mother plants $\left(P_{m}\right)$ is $30, d_{\text {runner }}=2.0, d_{\text {root }}=0.6$. In view of the conducted case studies on the benchmark functions, the proposed algorithm (rss-SBA) whose update equation is controlled by $\gamma$ is chosen as 4 . Further 15 independent trials are performed to pick the best one. All the computations are performed using MATLAB R2013a on a PC with Intel (R) core (TM) i5 CPU and 4.00 GB RAM.

\subsection{Discussion on results of linear antenna array}

In this type of configuration, the optimization is classified into two distinct categories:excitation amplitude control (position of antenna elements fixed) and position control (excitation amplitude chosen as Chebyshev with $-29 \mathrm{~dB}$ given in Fig. 13). Thus in excitation amplitude only control the problems investigated comprises of wide nulls, multiple nulls, and in position only control method single null and no null problems are considered.

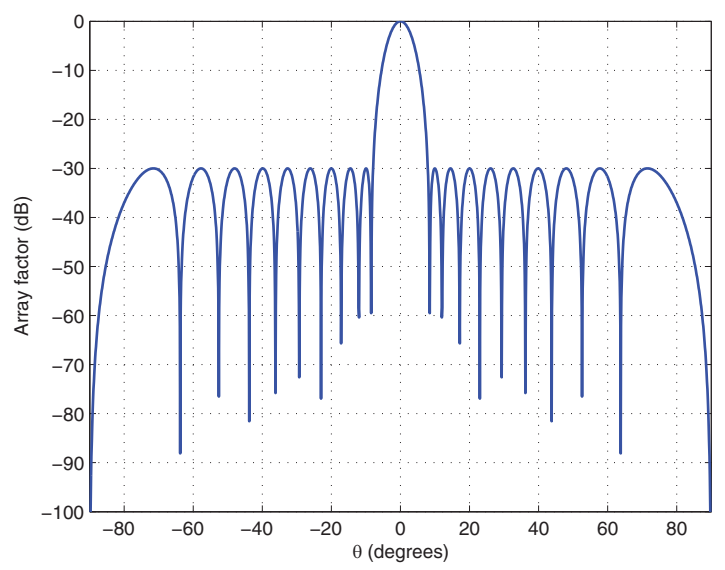

Figure 13. Chebyshev pattern for 20 elements (LAA) with $-29 \mathrm{~dB}$ masking envelope. 
TABLE 5. Optimized excitation amplitudes for 10 elements (LAA) with wide null centered at $30^{\circ}$ and width $5^{\circ}$.

\begin{tabular}{llllllllllllll}
\hline \hline $\begin{array}{l}\text { Optimization } \\
\text { method }\end{array}$ & & & \multicolumn{1}{c}{ Optimized amplitudes } & & & MSL (dB) & $\begin{array}{l}\text { NDL } \\
(\mathrm{dB})\end{array}$ \\
$\left(30^{\circ}\right)$
\end{tabular}

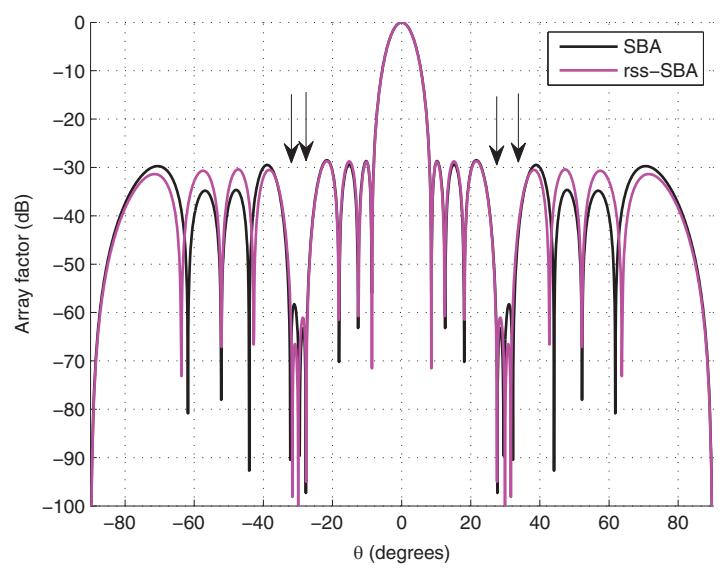

(a)

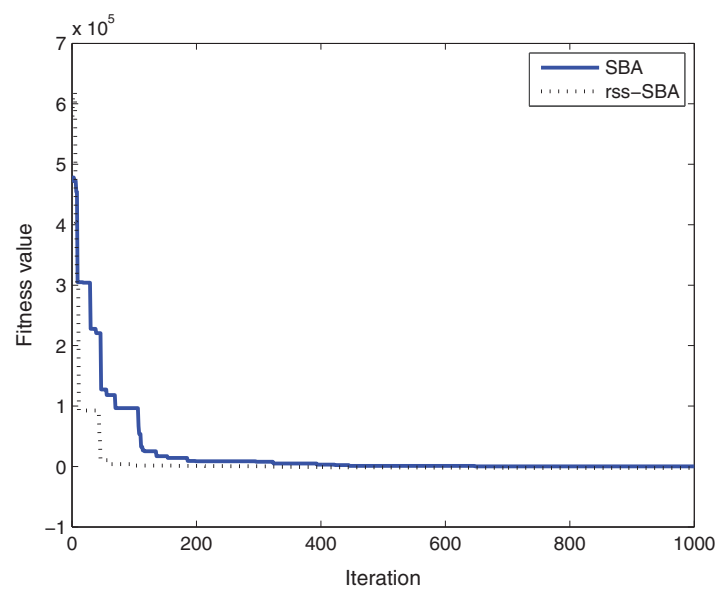

(b)

FiguRE 14. (a) Array pattern for 10 elements (LAA) with wide null centered at $30^{\circ}$ and width $5^{\circ}$.

(b) Convergence curve.

The main emphasis is given to plot the radiation pattern, convergence curve using SBA and rss-SBA and to determine the maximum sidelobe level (MSL) and null depth level (NDL) for each case study.

\section{Example 1 (10 elements) wide null centered around $30^{\circ}$ and width of $5^{\circ}$}

The results obtained are null level below $-60 \mathrm{~dB}$ and $-59 \mathrm{~dB}$ for proposed rss-SBA and conventional SBA respectively. The null at $30^{\circ}$ has crossed $-90 \mathrm{~dB}$. Table 5 shows the optimized current values along with MSL and NDL compared with BSA [15]. The radiation pattern is shown in the Figure 14a and convergence curve is shown in the Figure 14b.

\section{Example 2 (10 elements) wide null between $50^{\circ}$ and $60^{\circ}$}

The Null level using rss-SBA is below $-60 \mathrm{~dB}$ while that using SBA is below $-52 \mathrm{~dB}$. The radiation pattern and convergence characteristic are shown in the Figure 15 and the corresponding optimized excitation magnitudes are given in Table 6 . The null level using SMO [1] is reported below $-55 \mathrm{~dB}$.

\section{Example 3 (20 elements) Null at $14^{\circ}$}

MSL obtained is $-29.31 \mathrm{~dB}$ and NDL is $-129.4 \mathrm{~dB}$ using rss-SBA and MSL is $-29.31 \mathrm{~dB}$ and NDL is $-125.6 \mathrm{~dB}$ using SBA. Table 7 shows the optimized excitation amplitudes and corresponding MSL and NDL values compared with BSA [15]. The radiation pattern and the corresponding fitness curve is given in the Figure 16. 


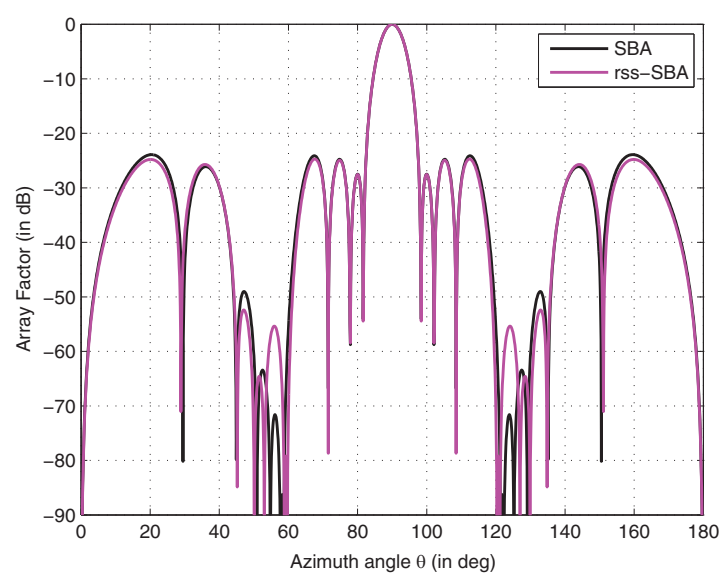

(a)

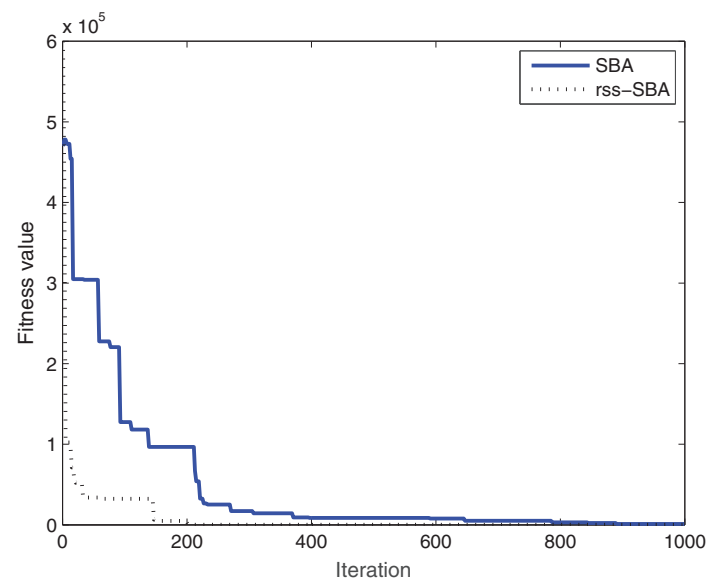

(b)

FIGURE 15. (a) Array pattern for 10 elements (LAA) with wide null centered around $40^{\circ}$ between $50^{\circ}$ and $60^{\circ}$. (b) Convergence curve.

TABLE 6. Optimized excitation amplitudes of 10 elements (LAA) for very wide null.

\begin{tabular}{lllllllllllll}
\hline \hline $\begin{array}{l}\text { Optimization } \\
\text { method }\end{array}$ & \multicolumn{10}{c}{ Optimized amplitudes } & & NDL (dB) \\
\hline SMO [1] & 0.772 & 0.771 & 0.773 & 0.645 & 0.496 & 0.505 & 0.368 & 0.461 & 0.199 & 0.166 & -55 \\
$\begin{array}{l}\text { SBA [29] } \\
\text { rss-SBA } \\
\text { (proposed) }\end{array}$ & 0.768 & 0.752 & 0.777 & 0.629 & 0.500 & 0.498 & 0.362 & 0.455 & 0.198 & 0.163 & -52 \\
\hline
\end{tabular}

TABLE 7. Optimized excitation amplitudes for 20 elements (LAA) with single null at $14^{\circ}$.

\begin{tabular}{|c|c|c|c|c|c|c|c|c|c|c|c|c|}
\hline \multicolumn{2}{|c|}{$\begin{array}{l}\text { Optimization } \\
\text { method }\end{array}$} & \multicolumn{9}{|c|}{ Optimized amplitudes } & \multirow{2}{*}{$\begin{array}{l}\text { MSL } \\
-29.22\end{array}$} & \multirow{2}{*}{$\begin{array}{l}\begin{array}{l}\text { NDL } \\
(\mathrm{dB})\end{array} \\
\left(14^{\circ}\right) \\
-134.2\end{array}$} \\
\hline $\mathrm{BSA}[15]$ & 1.000 & 0.98714 & 0.94987 & 0.88163 & 0.77212 & 0.63944 & 0.48298 & 0.33060 & 0.25882 & 0.29505 & & \\
\hline SBA [29] & 1.000 & 0.9878 & 0.95143 & 0.8807 & 0.77207 & 0.6383 & 0.4841 & 0.3369 & 0.2483 & 0.3012 & -29.56 & -125.6 \\
\hline rss-SBA & & & & & & & & & & & & \\
\hline (proposed) & 1.000 & 0.9826 & 0.9663 & 0.87665 & 0.76345 & 0.63452 & 0.4915 & 0.33655 & 0.25078 & 0.2831 & -29.89 & -134.2 \\
\hline
\end{tabular}

\section{Example 4 (20 elements) Nulls at $14^{\circ}$ and $26^{\circ}$}

In this example the NDL corresponding to $14^{\circ}$ and $26^{\circ}$ are $-135.2 \mathrm{~dB}$ and $-148.7 \mathrm{~dB}$ are respectively for rss-SBA while the same is $-135.2 \mathrm{~dB}$ and $-123.8 \mathrm{~dB}$ are for SBA respectively. The radiation pattern and the fitness curve for the double null objective is given in the Figure 17. The MSL is $-28.28 \mathrm{~dB}$ which is almost same for both SBA and rss-SBA. Table 8 shows the optimized excitation amplitudes and MSL and the NDL values are compared with BSA [15]. 


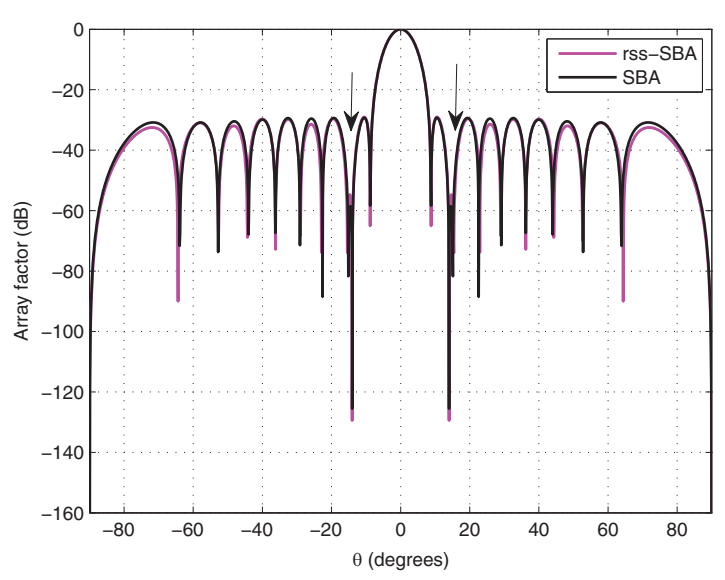

(a)

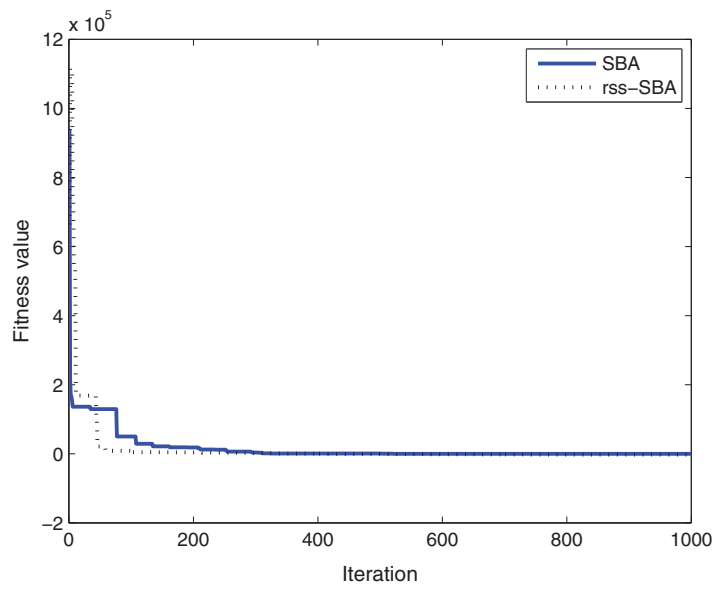

(b)

Figure 16. (a) Array pattern for 10 elements (LAA) with single null at $14^{\circ}$. (b) Convergence curve.

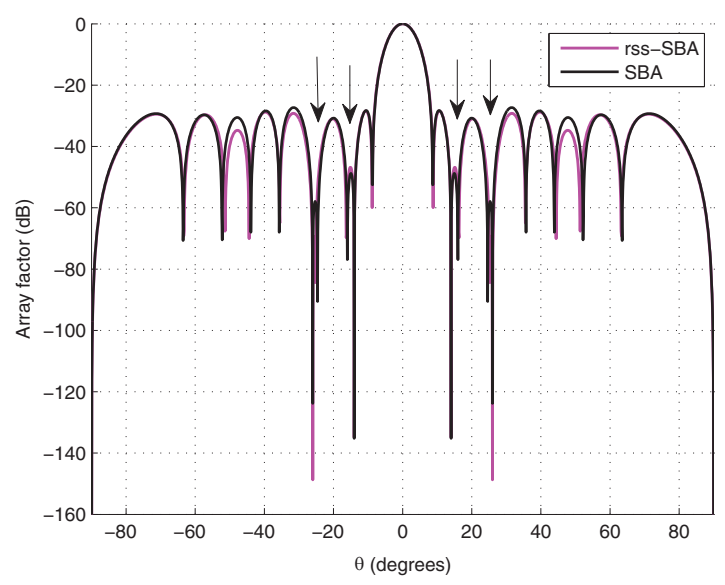

(a)

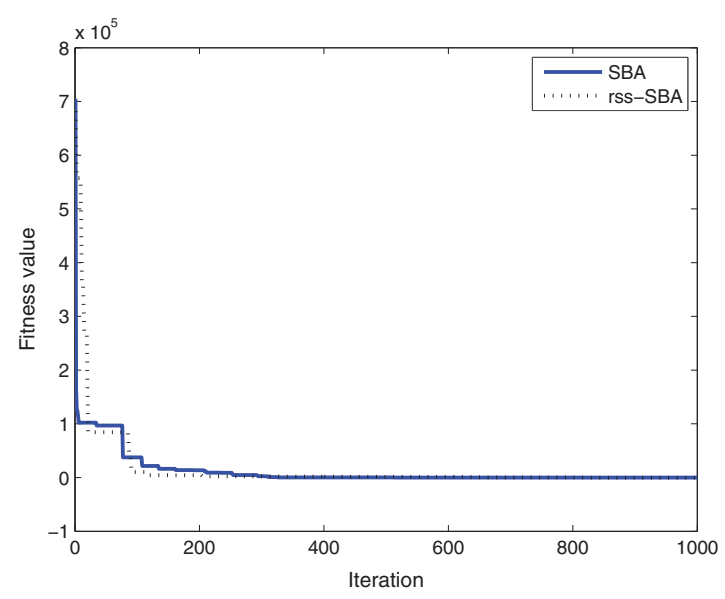

(b)

Figure 17. (a) Array pattern for 10 elements (LAA) with double null at $14^{\circ}$ and $26^{\circ}$. (b) Convergence curve.

TABLE 8. Optimized excitation amplitudes for 20 elements (LAA) with double null at $14^{\circ}$ and $26^{\circ}$.

\begin{tabular}{|c|c|c|c|c|c|c|c|c|c|c|c|c|c|}
\hline Optimization & & & & & Pptimized & amplitud & & & & & MSL & NDL & NDL \\
\hline $\mathrm{BSA}[15]$ & 0.8437 & 0.85439 & 0.85078 & 0.74231 & 0.6098 & 0.5508 & 0.44059 & 0.3053 & 0.17434 & 0.2239 & -28.47 & -131.2 & -130.7 \\
\hline SBA [29] & 0.9666 & 1.0000 & 0.99216 & 0.8648 & 0.7162 & 0.6405 & 0.5251 & 0.35759 & 0.2018 & 0.2871 & -28.28 & -135.2 & -123.8 \\
\hline $\begin{array}{l}\text { rss-SBA } \\
\text { (proposed) }\end{array}$ & 0.9834 & 0.9824 & 1.000 & 0.8660 & 0.71059 & 0.64107 & 0.50660 & 0.36585 & 0.20344 & 0.26316 & -28.28 & -135.2 & -148.7 \\
\hline
\end{tabular}


TABLE 9. Optimized excitation amplitudes for 20 elements (LAA) with triple nulls at $14^{\circ}, 26^{\circ}$ and $33^{\circ}$.

\begin{tabular}{|c|c|c|c|c|c|c|c|c|c|c|c|c|c|c|}
\hline Optimization & & & & & timized a & amplitude & & & & & MSL & NDL & NDL & NDL \\
\hline $\mathrm{BSA}[15]$ & 0.9875 & 1.000 & 0.9900 & 0.8505 & 0.7459 & 0.60333 & 0.3613 & 0.46506 & 0.2065 & 0.1512 & -27.24 & -126.8 & -119.1 & -120.2 \\
\hline SBA [29] & 1.000 & 0.9873 & 0.9916 & 0.90004 & 0.75541 & 0.6133 & 0.4722 & 0.4509 & 0.2055 & 0.2188 & -27.16 & -110.8 & -109.7 & -128.2 \\
\hline $\begin{array}{l}\text { rss-SBA } \\
\text { (proposed) }\end{array}$ & 1.000 & 0.99266 & 0.986 & 0.8860 & 0.7470 & 0.5966 & 0.4487 & 0.4313 & 0.2116 & 0.1900 & -28.68 & -134.3 & -122.5 & -134.7 \\
\hline
\end{tabular}

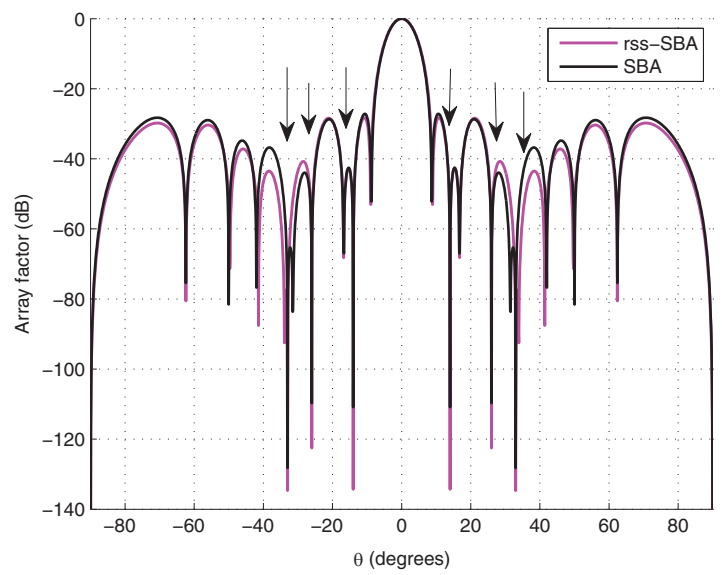

(a)

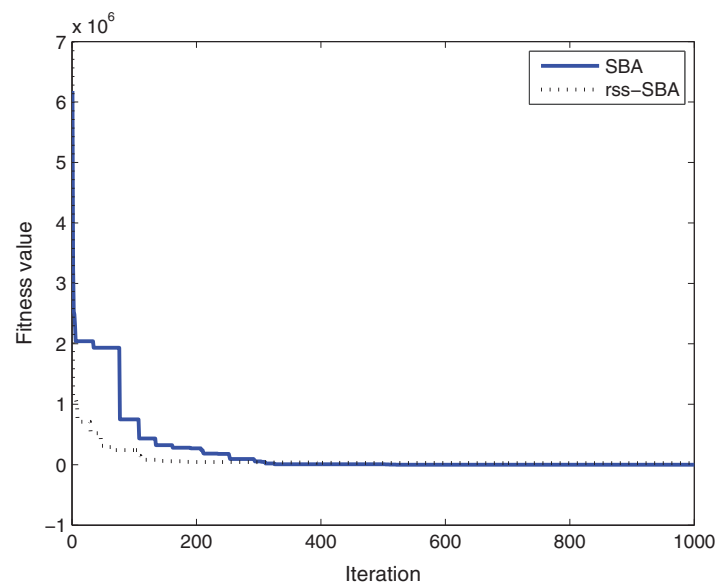

(b)

Figure 18. (a) Array pattern for 20 elements (LAA) with triple null at $14^{\circ}, 26^{\circ}$ and $33^{\circ}$. (b) Convergence curve.

\section{Example 5 (20 elements) nulls at $14^{\circ}, 26^{\circ}$ and $33^{\circ}$}

The NDL values obtained at the nulls $14^{\circ}, 26^{\circ}$ and $33^{\circ}$ are $-134.3 \mathrm{~dB},-122.5 \mathrm{~dB}$ and $-134.7 \mathrm{~dB}$, respectively for rss-SBA and $-110.8 \mathrm{~dB},-109.7 \mathrm{~dB}$ and $-128.2 \mathrm{~dB}$ for SBA respectively. The MSL obtained is $-28.68 \mathrm{~dB}$ employing rss-SBA and $-27.16 \mathrm{~dB}$ using SBA. Table 9 shows the optimized excitation amplitude values along with MSL and NDL for different algorithms. The radiation pattern and the corresponding fitness curve is given in the Figure 18.

\section{Example 6 (32 elements single null at $\left.99^{\circ}\right)$}

Another experiment was carried out which is different from previous five examples where position only control mode is considered to prove the consistency of the proposed rss-SBA technique to effectively handle varieties of the problem in antenna design domain. Here a 32 element linear array is considered where the prescribed null is at $\theta=81^{\circ}$ (and $\theta=99^{\circ}$, the mirror image with reference to $y$-axis at $90^{\circ}$ taking a symmetrical configuration). In addition to the usual objective of minimizing the MSL in the entire scanning angle $\left(\theta=0^{\circ}-180^{\circ}\right)$ leaving apart the main lobe $\left(\theta=84^{\circ}-96^{\circ}\right)$. The performance results have been quite significant in the case of rss-SBA in comparison to CSO [36] and ALO [43]. Table 10 summarizes the optimized locations using different algorithms while the antenna elements are excited uniformly. The array pattern shown in the Figure 19 is self explanatory and clearly speaks about the highly encouraging results obtained using rss-SBA in terms of remarkable improvement in MSL $(-20.80 \mathrm{~dB})$ and NDL $(-93.49 \mathrm{~dB})$ when compared to the previous best contenders ALO [43], winning by a wide margin. 
TABLE 10. Optimized position of $N=32$ elements of (LAA) with single null.

\begin{tabular}{|c|c|c|c|c|c|c|c|c|c|c|}
\hline \multicolumn{3}{|c|}{$\begin{array}{l}\text { Optimization } \\
\text { method }\end{array}$} & \multicolumn{6}{|c|}{ Optimized element positions } & \multirow{2}{*}{$\begin{array}{l}\text { MSL } \\
(\mathrm{dB})\end{array}$} & \multirow{2}{*}{$\begin{array}{l}\text { NDL } \\
(\mathrm{dB}) \\
\left(99^{\circ}\right) \\
-93.49\end{array}$} \\
\hline & 0.2305 & 0.8476 & 1.5532 & 2.3411 & 3.1724 & 4.1019 & 5.0681 & 6.3408 & & \\
\hline (prop & 0.50290 & 1.1981 & 1.9374 & 2.7519 & 3.6280 & 4.5768 & 5.6885 & 7.0268 & & \\
\hline \multirow{2}{*}{ SBA } & 0.2565 & & 1.9057 & 2.5814 & 3.3475 & 4.33 & 5.5538 & 7.0814 & \multirow{2}{*}{-19.23} & \multirow[t]{2}{*}{-88.08} \\
\hline & & & 2.41 & & & 4.9 & & 7.86 & & \\
\hline \multirow[t]{2}{*}{ CSO [36] } & 0.2883 & 1.1929 & 1.9768 & 2.6886 & 3.4848 & 4.3822 & 5.4817 & 7.0412 & \multirow[t]{2}{*}{-18.24} & \multirow[t]{2}{*}{-80} \\
\hline & 0.6830 & 1.5199 & 2.3247 & 3.1362 & 3.9538 & 4.9252 & 6.2091 & 7.7500 & & \\
\hline \multirow[t]{2}{*}{ ALO [43] } & 0.2121 & 1.2499 & 2.2355 & 3.0146 & 3.7627 & 4.7500 & 5.7500 & 7.2500 & \multirow[t]{2}{*}{-15.5} & \multirow[t]{2}{*}{-83.65} \\
\hline & 0.7412 & 1.7500 & 2.6938 & 3.4513 & 4.2684 & 5.2532 & 6.4509 & 7.9990 & & \\
\hline
\end{tabular}

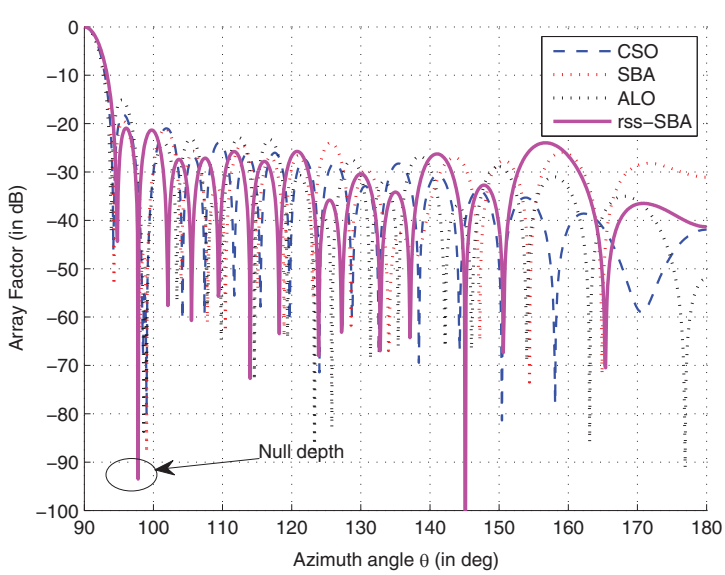

(a)

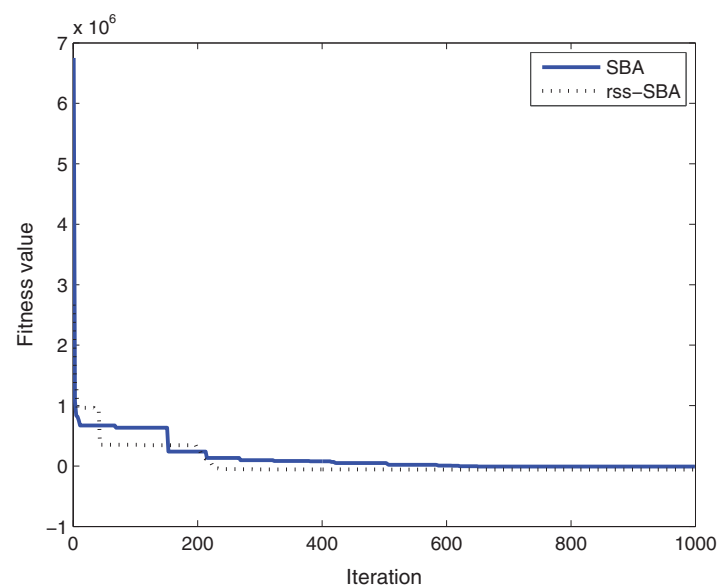

(b)

Figure 19. (a) Array pattern for 32 elements (LAA) with single null at 99. (b) Convergence curve.

\section{Example 7 (10 elements with no nulls specified)}

One more experiment is conducted in the position only control mode where no nulls are specified and the MSL is only minimized. The radiation pattern is shown in Figure 20 along with the convergence characteristics. Table 11 shows the optimized positions along with MSL for both SBA and rss-SBA and compared with HSA [17].

\subsection{Discussion on results of circular antenna array}

In order to encompass the domain of application to a further level, the circular array synthesis is brought into its ambit. Both the optimized element locations and their amplitudes have been determined considering the aperture size and side lobe level for an antenna array with $N=10$ elements. The application of the proposed algorithm has witnessed an encouraging result for this case study also and there by consolidates the pertinent issues that Strawberry algorithm as well as the proposed rss-SBA applied to optimization can definitely contribute to performance gain. Figure 21 shows the radiation patterns as well as convergence characteristic for the circular configuration considered. Table 12 depicts the position of antenna elements and their excitations both using PSO [24] and the SBA as well as the proposed rss-SBA. It is quite evident from Table 13 that the proposed SBA has outperformed the PSO [24] in terms of beam width and maximum sidelobe level. 


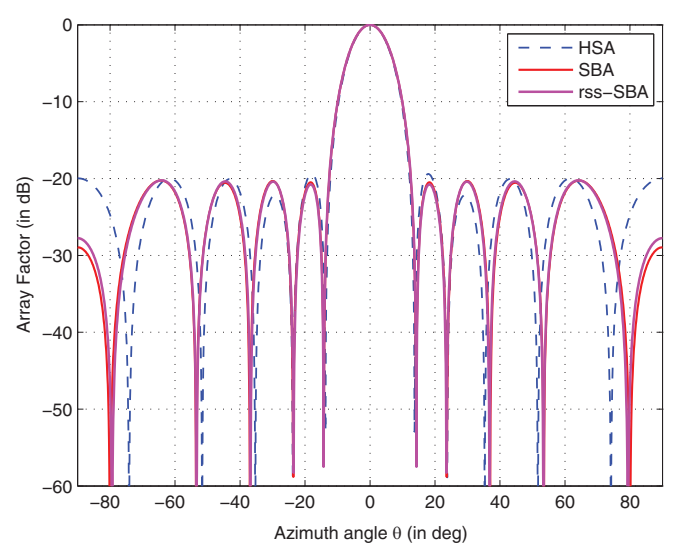

(a)

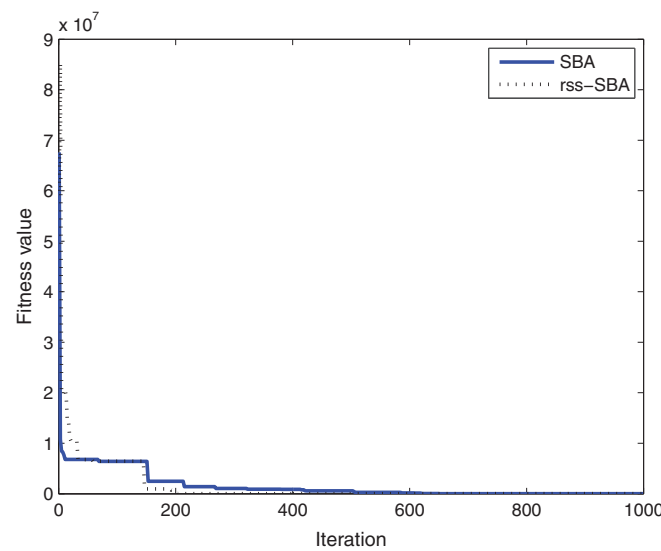

(b)

Figure 20. (a) Array pattern for 10 elements (LAA) with no nulls specified. (b) Convergence curve.

TABLE 11. Optimized positions of 10 element (LAA) with no nulls specified.

\begin{tabular}{lllllll}
\hline \hline Optimization method & \multicolumn{5}{c}{ Optimized element positions } & MSL (dB) \\
\hline HSA [17] & 0.497 & 1.122 & 2.088 & 3.000 & 4.350 & -19.41 \\
SBA [29] & 0.4720 & 1.0560 & 2.0140 & 2.9420 & 4.2520 & -20.33 \\
rss-SBA (proposed) & 0.4760 & 1.0500 & 2.0080 & 2.9380 & 4.2580 & -20.37 \\
\hline
\end{tabular}

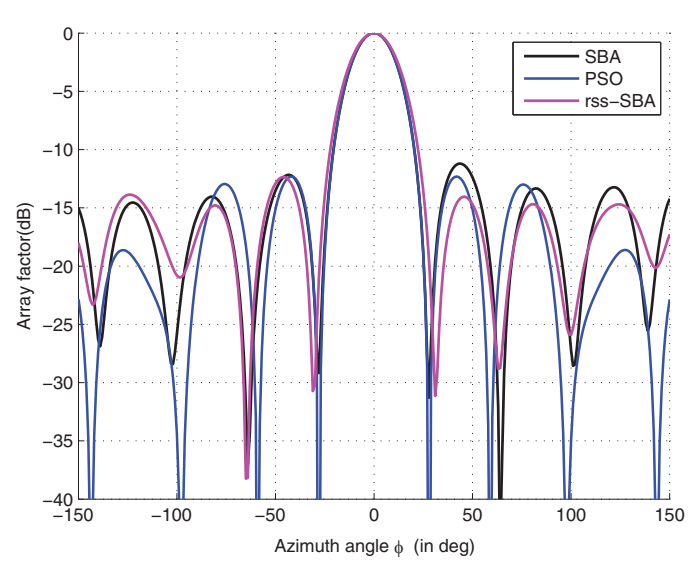

(a)

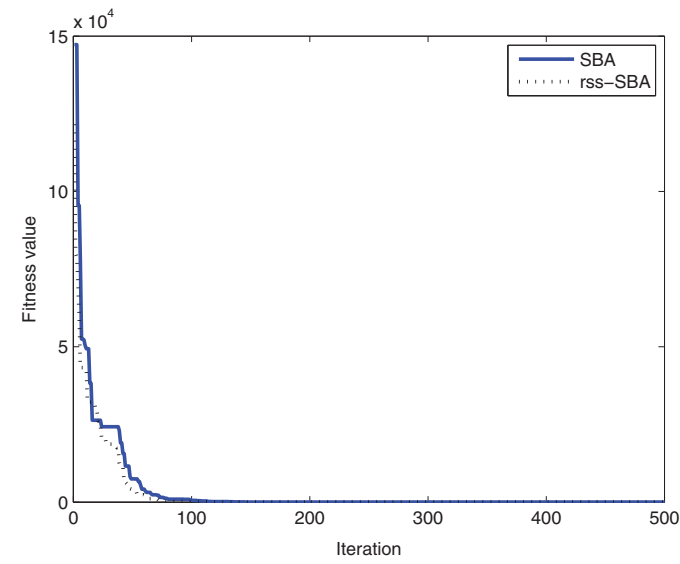

(b)

Figure 21. (a) Array pattern for 10 elements (CAA). (b) Convergence curve.

So far as the parameter selection of SBA are concerned, $P_{m}=30$, Itmax $=500$ and 15 independent trials are chosen. The objective function constants $c_{1}, c_{2}$ and $c_{3}$ are set as 0.4, 0.2 and 0.4, respectively. The other algorithm parameters are set as $d_{\text {runner }}=2.0, d_{\text {root }}=0.6$, and $\gamma=4$. The performance indices $i . e$. the maximum sidelobe level, the beam width and the minimum circumference $(\mathrm{kr})$ obtained by applying the proposed rss-SBA algorithm are $-13.29 \mathrm{~dB}, 22.40^{\circ}$ and $5.9197 \lambda$ respectively. The comparison is made with PSO [24] having the corresponding values as $-12.3 \mathrm{~dB}, 24.34^{\circ}$ and $5.9196 \lambda$. 
TABLE 12. Optimized excitation amplitudes and positions of 10 elements (CAA).

\begin{tabular}{|c|c|c|c|c|c|c|c|c|c|c|}
\hline \multicolumn{5}{|c|}{$\begin{array}{l}\text { Optimization } \\
\text { method }\end{array}$} & \multicolumn{6}{|c|}{ rss-SBA (proposed) } \\
\hline $\begin{array}{l}\text { Excitation } \\
\text { amplitude }\end{array}$ & 0.9942 & 0.7652 & 0.5771 & 0.9561 & 0.7015 & 1.000 & 0.5281 & 0.7994 & 0.9791 & 0.9792 \\
\hline Positions & 0.3021 & 0.9678 & 0.4206 & 0.9449 & 0.3068 & 0.3508 & 0.9389 & 0.4042 & 0.9536 & 0.3299 \\
\hline \multicolumn{5}{|c|}{$\begin{array}{l}\text { Optimization } \\
\text { method }\end{array}$} & \multicolumn{3}{|c|}{ SBA $[29]$} & & & \\
\hline $\begin{array}{l}\text { Excitation } \\
\text { amplitude }\end{array}$ & 0.6325 & 0.6742 & 0.6266 & 0.6170 & 0.5769 & 0.5770 & 0.6170 & 0.6266 & 0.6742 & 0.8845 \\
\hline Positions & 0.3386 & 0.9286 & 0.4258 & 0.9382 & 0.3178 & 0.3179 & 0.9382 & 0.4258 & 0.9286 & 0.3386 \\
\hline $\begin{array}{l}\text { Optimizatio } \\
\text { method }\end{array}$ & & & & & PSO & {$[24]$} & & & & \\
\hline $\begin{array}{l}\text { Excitation } \\
\text { amplitude }\end{array}$ & 1.0000 & 0.7529 & 0.7519 & 1.0000 & 0.5062 & 1.0000 & 0.7501 & 0.7524 & 1.0000 & 0.5067 \\
\hline Positions & 0.3170 & 0.9654 & 0.3859 & 0.9654 & 0.3185 & 0.3164 & 0.9657 & 0.3862 & 0.9650 & 0.3174 \\
\hline
\end{tabular}

TABLE 13. Comparative Analysis for CAA design.

\begin{tabular}{llll}
\hline \hline Optimization method & SLL $(\mathrm{dB})$ & Beam width $(\mathrm{deg})$ & Circumference $(k r)$ \\
\hline rss-SBA (proposed) & -13.29 & 24.4 & $5.9196 \lambda$ \\
SBA & -11.53 & 24.1 & $5.8981 \lambda$ \\
PSO [24] & -12.3 & 24.34 & $5.9029 \lambda$ \\
\hline
\end{tabular}

\section{Conclusion}

The present work comprises two independent contributions. In the first part the conventional SBA is applied to antenna array synthesis problems for the first time and the performance study has been done in comparison with other metaheuristic algorithms reported in the literature. The SBA has successfully derives very promising results both for linear and circular array configurations. In the second part a completely novel algorithm is developed based on the original SBA but a new variant of it has been evolved where the length or step size $d_{\text {runner }}$ and $d_{\text {root }}$ have been continually reduced while the optimization process (rather keeping them fixed as in case of conventional SBA) is progressing. This basically aims at the objective that the search space shrinks when the solution vector is reaching towards the optima and thus necessitating the need of reducing those step length of $d_{\text {runner }}$ and $d_{\text {root }}$ to ensure global convergence else the solution may stuck to premature convergence yielding local optima. The proposed algorithm known as reduced step size SBA (rss-SBA) has been applied to many standard benchmark functions. The results are quite inspiring. Then attempt has been made to further explore its application to practical design problem and the same is accomplished by chosing antenna array synthesis issues both in linear and circular array configurations. The performances have been quite praise worthy surpassing all expectations and the proposed rss-SBA has come out as a clear winner in all competitions in the optimization domain.

\section{REFERENCES}

[1] A.A. Al-Azza, A.A. Al-Jodah and F.J. Harackiewicz, Spider monkey optimization: a novel technique for antenna optimization. IEEE Antennas Wirel. Propag. Lett. 15 (2016) 1016-1019.

[2] F.J. Ares-Pena, J.A. Rodriguez-Gonzalez, E. Villanueva-Lopez and S. Rengarajan, Genetic algorithms in the design and optimization of antenna array patterns. IEEE Trans. Antennas Propag. 47 (1999) 506-510. 
[3] B. Babayigit and R. Ozdemir, Design of non-uniform circular antenna using a modified abc algorithm. In: Electrical, Electronic and Computer Engineering Symposium. Bursa, (2012) 238-241.

[4] B. Babayigit, A. Akdagli and K. Guney, A clonal selection algorithm for null synthesizing of linear antenna arrays by amplitude control. J. Electromagnet. Waves App. 20 (2006) 1007-1020.

[5] C.A. Balanis, Antenna Theory: Analysis and Design. John Wiley \& Sons, New York, NY (2012).

[6] Y. Cengiz and H. Tokat, Linear antenna array design with use of genetic, memetic and tabu search optimization algorithms. Prog. Electromagnet. Res. C 1 (2008) 63-72.

[7] B.S. Charan, A. Mittal and R. Tiwari, Multirobot navigation in unknown environment using strawberry algorithm. Int. J. Rob. App. Technol. (IJRAT) 5 (2017) 63-81.

[8] I. Chidambaram and R. Francis, Automatic generation control of a two area reheat interconnected power system based on cps using fuzzy neural network. In: 2011 International Conference on Emerging Trends in Electrical and Computer Technology (ICETECT). IEEE (2011) 200-205.

[9] Y.C. Chung and R.L. Haupt, Amplitude and phase adaptive nulling with a genetic algorithm. J. Electromagnet. Waves App. 14 (2000) 631-649.

[10] P. Civicioglu, Backtracking search optimization algorithm for numerical optimization problems. Appl. Math. Comput. 219 (2013) 8121-8144.

[11] N.I. Dib, S.K. Goudos and H. Muhsen, Application of taguchi's optimization method and self-adaptive differential evolution to the synthesis of linear antenna arrays. Prog. Electromagnet. Res. 102 (2010) 159-180.

[12] S.K. Goudos, V. Moysiadou, T. Samaras, K. Siakavara and J.N. Sahalos, Application of a comprehensive learning particle swarm optimizer to unequally spaced linear array synthesis with sidelobe level suppression and null control. IEEE Antennas Wirel. Propag. Lett. 9 (2010) 125-129.

[13] K. Guney and S. Basbug, Interference suppression of linear antenna arrays by amplitude-only control using a bacterial foraging algorithm. Prog. Electromagnet. Res. 79 (2008) 475-497.

[14] K. Guney and S. Basbug, Seeker optimization algorithm for interference suppression of linear antenna arrays by controlling position-only, phase-only, and amplitude-only. Int. J. RF Microwave Comput. Aided Eng. 21 (2011) 505-518.

[15] K. Guney and A. Durmus, Pattern nulling of linear antenna arrays using backtracking search optimization algorithm. Int. J. Antennas Propag. 2015 (2015) 713080.

[16] K. Guney and M. Onay, Amplitude-only pattern nulling of linear antenna arrays with the use of bees algorithm. Prog. Electromagnet. Res. 70 (2007) 21-36.

[17] K. Guney and M. Onay, Optimal synthesis of linear antenna arrays using a harmony search algorithm. Expert Syst. App. 38 (2011) 15455-15462.

[18] K. Guney, A. Durmus and S. Basbug, A plant growth simulation algorithm for pattern nulling of linear antenna arrays by amplitude control. Prog. Electromagnet. Res. B 17 (2009) 69-84.

[19] K. Guney, A. Durmus and S. Basbug, Backtracking search optimization algorithm for synthesis of concentric circular antenna arrays. Int. J. Antennas Propag. 2014 (2014) 250841.

[20] N. Karaboga, K. Güney and A. Akdagli, Null steering of linear antenna arrays with use of modified touring ant colony optimization algorithm. Int. J. RF Microwave Comput. Aided Eng. 12 (2002) 375-383.

[21] S. Karimkashi and A.A. Kishk, Invasive weed optimization and its features in electromagnetics. IEEE Trans. Antennas Propag. 58 (2010) 1269-1278.

[22] H.N. Khan, H. Iftikhar, S. Asif, R. Maroof, K. Ambreen and N. Javaid, Demand side management using strawberry algorithm and bacterial foraging optimization algorithm in smart grid. In: International Conference on Network-Based Information Systems. Springer (2017) 191-202.

[23] M.S. Khan, C.A. ul Hassan, H.A. Sadiq, I. Ali, A. Rauf and N. Javaid, A new meta-heuristic optimization algorithm inspired from strawberry plant for demand side management in smart grid. In: International Conference on Intelligent Networking and Collaborative Systems. Springer, Berlin-Heidelberg (2017) 143-154.

[24] M.M. Khodier and M. Al-Aqeel, Linear and circular array optimization: a study using particle swarm intelligence. Prog. Electromagnet. Res. B 15 (2009) 347-373.

[25] M.M. Khodier and C.G. Christodoulou, Linear array geometry synthesis with minimum sidelobe level and null control using particle swarm optimization. IEEE Trans. Antennas Propag. 53 (2005) 2674-2679.

[26] X. Li and G. Yang, Artificial bee colony algorithm with memory Appl. Soft Comput. 41 (2016) 362-372.

[27] J. Liang, B. Qu and P. Suganthan, Problem definitions and evaluation criteria for the CEC 2014 special session and competition on single objective real-parameter numerical optimization. Computational Intelligence Laboratory, Zhengzhou University, Zhengzhou China and Technical Report, Nanyang Technological University, Singapore (2013).

[28] W.-P. Liao, Array pattern synthesis with null steering using genetic algorithms by controlling only the current amplitudes. Int. J. Electron. 86 (1999) 445-457.

[29] F. Merrikh-Bayat, A numerical optimization algorithm inspired by the strawberry plant. Preprint arXiv:1407.7399.

[30] S. Mirjalili, The ant lion optimizer. Adv. Eng. Softw. 83 (2015) 80-98.

[31] S. Mirjalili, S.M. Mirjalili and A. Hatamlou, Multi-verse optimizer: a nature-inspired algorithm for global optimization. Neural Comput. App. 27 (2016) 495-513.

[32] S. Pal, A. Basak and S. Das, Linear antenna array synthesis with modified invasive weed optimisation algorithm. Int. J. Bio-Inspired Comput. 3 (2011) 238-251. 
[33] M.A. Panduro, A.L. Mendez, R. Dominguez and G. Romero, Design of non-uniform circular antenna arrays for side lobe reduction using the method of genetic algorithms. AEU-Int. J. Electron. Commun. 60 (2006) 713-717.

[34] L. Pappula and D. Ghosh, Constraint-based synthesis of linear antenna array using modified invasive weed optimization. Prog. Electromagnet. Res. M 36 (2014) 9-22.

[35] L. Pappula and D. Ghosh, Large array synthesis using invasive weed optimization. In: 2013 International Conference on Microwave and Photonics (ICMAP). IEEE (2013) 1-6.

[36] L. Pappula and D. Ghosh, Linear antenna array synthesis using cat swarm optimization. AEU-Int. J. Electron. Commun. 68 (2014) 540-549.

[37] G. Ram, D. Mandal, R. Kar and S.P. Ghoshal, Circular and concentric circular antenna array synthesis using cat swarm optimization. IETE Tech. Rev. 32 (2015) 204-217.

[38] E. Rajo-Iglesias and O. Quevedo-Teruel, Linear array synthesis using an ant-colony-optimization-based algorithm. IEEE Antennas Propag. Mag. 49 (2007) 70-79.

[39] M. Rattan, M. Patterh and B. Sohi, Optimization of circular antenna arrays of isotropic radiators using simulated annealing. Int. J. Microwave Wirel. Technol. 1 (2009) 441.

[40] A. Recioui, Sidelobe level reduction in linear array pattern synthesis using particle swarm optimization. J. Optim. Theory App. 153 (2012) 497-512.

[41] G.G. Roy, S. Das, P. Chakraborty and P.N. Suganthan, Design of non-uniform circular antenna arrays using a modified invasive weed optimization algorithm. IEEE Trans. Antennas Propag. 59 (2011) 110-118.

[42] P. Saxena and A. Kothari, Linear antenna array optimization using flower pollination algorithm. SpringerPlus 5 (2016) 1.

[43] P. Saxena and A. Kothari, Ant lion optimization algorithm to control side lobe level and null depths in linear antenna arrays. AEU-Int. J. Electron. Commun. 70 (2016) 1339-1349.

[44] P. Saxena and A. Kothari, Optimal pattern synthesis of linear antenna array using grey wolf optimization algorithm. Int. J. Antennas Propag. 2016 (2016) 1205970.

[45] A. Sharaqa and N. Dib, Circular antenna array synthesis using firefly algorithm. Int. J. RF Microwave Comput. Aided Eng. 24 (2014) 139-146.

[46] U. Singh and T. Kamal, Design of non-uniform circular antenna arrays using biogeography-based optimisation. IET Microwaves Antennas Propag. 5 (2011) 1365-1370.

[47] U. Singh, H. Kumar and T.S. Kamal, Linear array synthesis using biogeography based optimization. Prog. Electromagnet. Res. M 11 (2010) 25-36.

[48] U. Singh and M. Rattan, Design of linear and circular antenna arrays using cuckoo optimization algorithm. Prog. Electromagnet. Res. C 46 (2014) 1-11.

[49] U. Singh and R. Salgotra, Optimal synthesis of linear antenna arrays using modified spider monkey optimization. Arabian J. Sci. Eng. 41 (2016) 1-17.

[50] S. Yang, Y.B. Gan and A. Qing, Antenna-array pattern nulling using a differential evolution algorithm. Int. J. RF Microwave Comput.-Aided Eng. 14 (2004) 57-63.

[51] M.A. Zaman and A. Matin, Nonuniformly spaced linear antenna array design using firefly algorithm. Int. J. Microwave Sci. Technol. 2012 (2012) 256759.

[52] Z. Zhang, T. Li, F. Yuan and L. Yin, Synthesis of linear antenna array using genetic algorithm to control side lobe level. In: Computer Engineering and Networking. Springer, Berlin-Heidelberg (2014) 39-46. 